\title{
Modelling of Solar Radiation Pressure Effects: Parameter Analysis for the MICROSCOPE Mission
}

\author{
Meike List, Stefanie Bremer, Benny Rievers, and Hanns Selig
}

ZARM, University of Bremen, Am Fallturm, 28359 Bremen, Germany

Correspondence should be addressed to Meike List; meike.list@zarm.uni-bremen.de

Received 30 July 2015; Accepted 19 October 2015

Academic Editor: Paolo Tortora

Copyright (C) 2015 Meike List et al. This is an open access article distributed under the Creative Commons Attribution License, which permits unrestricted use, distribution, and reproduction in any medium, provided the original work is properly cited.

\begin{abstract}
Modern scientific space missions pose high requirements on the accuracy of the prediction and the analysis of satellite motion. On the one hand, accurate orbit propagation models are needed for the design and the preparation of a mission. On the other hand, these models are needed for the mission data analysis itself, thus allowing for the identification of unexpected disturbances, couplings, and noises which may affect the scientific signals. We present a numerical approach for Solar Radiation Pressure modelling, which is one of the main contributors for nongravitational disturbances for Earth orbiting satellites. The here introduced modelling approach allows for the inclusion of detailed spacecraft geometries, optical surface properties, and the variation of these optical surface properties (material degradation) during the mission lifetime. By using the geometry definition, surface property definitions, and mission definition of the French MICROSCOPE mission we highlight the benefit of an accurate Solar Radiation Pressure modelling versus conventional methods such as the Cannonball model or a Wing-Box approach. Our analysis shows that the implementation of a detailed satellite geometry and the consideration of changing surface properties allow for the detection of systematics which are not detectable by conventional models.
\end{abstract}

\section{Introduction}

The modelling and propagation of satellite motion are one of the central tasks in mission analysis. The main driver for the evolution of a satellite orbit is the gravitational field of the central attracting mass. While a spherical symmetric approach for the gravitational field delivers undisturbed Kepler orbits, more realistic approaches employ spherical harmonics to model the gravitational potential. Among others, these models implement the effect of Earth oblateness, zonal, and tesseral variations of the mass distribution. Consequently, the introduced corrections of the gravitational field can be interpreted as a gravitational disturbance of an ideal Kepler orbit.

However, besides these perturbations, nongravitational disturbance (NGD) effects have a large influence on satellite motion. The largest of these NGDs in low orbit altitudes is the atmospheric drag resulting from the resistance of residual atmosphere against the satellite body moving at high relative speed. For higher altitudes, where the influence of residual atmosphere can be neglected, the dominant NGDs result from interaction of the satellite surface with solar photons, causing a drag force known as the Solar Radiation Pressure (SRP). The magnitude of the SRP acting on the satellite depends on a wide range of parameters. The distance to the Sun and the position of the satellite with respect to Earth and Sun (regarding possible eclipses) define the intensity of the incoming radiation. The geometry of the satellite, the optical properties of the external surfaces, and the actual orientation with respect to the Sun largely influence the orientation and magnitude of the evolving SRP. According to this, any SRP model depends on an accurate implementation of the satellite orbit, the attitude, and the geometric/physical properties of the satellite structure. As a consequence, a high modelling effort has to be made in order to obtain precise results. However, if mission planning and analysis for the satellite mission at hand possess high requirements on orbit modelling precision, a sophisticated SRP model is needed.

It has been argued for quite some time that commonly used SRP models like the Cannonball and the Wing-Box model are not sufficient enough for an accurate SRP analysis $[1,2]$. This is particularly true if the involved geometries differ considerably from a spherical shape or a standard bus and 
solar panel assembly. The high gain in modelling accuracy by means of a realistic implementation of the satellite geometry has also been demonstrated with an analysis of NGDs acting during the cruise phases of the ESA Rosetta spacecraft [3]. Here a nonphysical solar constant was measured resulting from a parametric fit of the measured contribution of SRP on the total acceleration. By means of a sophisticated SRP and thermal radiation pressure (TRP) (TRP results from photons emitted by the spacecraft itself) model this offset was explained as a nonmodeled TRP correlated with the acting SRP. Further examples for a successful implementation of enhanced SRP models are GNSS satellites, where navigation accuracy directly benefits from an improved SRP modelling approach [4-6].

The modelling effort for an accurate analysis of the SRP effect on a given satellite is considerably high. Consequently, a trade-off has to be made between the precision requirements for the specific mission, the effort that one is willing to take, and the possible gain with respect to an improvement of a precise implementation of NGDs. This paper intends to give an overview on the implications of accurate SRP modelling and the expected improvement of NGD implementation. The parameters for the subsequent SRP analysis are derived from the French space mission MICROSCOPE [7] which delivers a suitable test case with respect to the specified mission profile.

The MICROSCOPE mission requires a very high accuracy of the spacecraft attitude and attitude stability due to the specific mission specification. In order to realize the high performance of the differential acceleration measurement of the two test masses to test the Weak Equivalence Principle $(\mathrm{EP})$ it is essential to ensure a very low disturbance level (forces and torques acting on the satellite). For this purpose MICROSCOPE will be operated in drag-free mode. Any disturbance will be compensated by forces and torques generated by a cold gas propulsion system in closed loop control. The input to the corresponding controller is given by the common mode acceleration signal of the differential accelerometer while the science signal is extracted from the differential acceleration signal. However, in spite of the drag-free control, the exact modelling of NGDs is still necessary due to couplings between the accelerometers and the satellite structure. As a consequence, external disturbance effects influence the scientific signals since the drag-free control forces and torques needed to compensate the NGDs introduce a disturbance translated by the coupling.

The actual requirements of MICROSCOPE are quite demanding. For the EP measurement sessions the residual acceleration of the spacecraft shall be less than $10^{-12} \mathrm{~ms}^{-2}$. At the EP test frequency, the angular pointing stability should be better than $7 \mu \mathrm{rad}$, and the angular velocity stability is required to not exceed $10^{-9} \mathrm{rads}^{-1}$, respectively [8].

The sun-synchronous polar MICROSCOPE orbit leads to a force vector due to SRP always directed to one side of the orbital plane. This leads to a linear acceleration normal to the orbital plane which is superimposed by an angular variation due to seasonal change of the angle between the orbital plane normal and the direction to the sun. In case of simulating a drag-free mission a detailed modelling of the corresponding SRP forces and torques is important to estimate the actual control forces of the Attitude and Orbit Control System (AOCS) which keep the spacecraft in the favored state. Considering MICROSCOPE, NGD effects due to SRP can easily reach several $\mu \mathrm{N}$. Divided by the satellite's mass $(330 \mathrm{~kg})$ this force induces disturbing accelerations of some $10^{-8} \mathrm{~ms}^{-2}$ which is not negligible in a premission endto-end simulation and for developing and implementing data analysis and data processing strategies.

Due to the high demands of the mission, the sunsynchronous orbital plane, and the LEO character of the orbit, MICROSCOPE is an ideal test case scenario for the analysis of the general benefit of accurate SRP modelling for space missions. After a general introduction of the SRP modelling method and the derivation of SRP characteristics for chosen orbit and mission examples we will use MICROSCOPE as a test case scenario for a detailed SRP analysis. By looking at different approaches for the implementation of the geometry of the satellite and applying surface degradation models we highlight the possible benefits and the involved costs of high accuracy SRP modelling.

\section{Orbit and Attitude Propagation}

Since the evolving SRP magnitude and orientation depend on the position and the attitude of the spacecraft, a dynamic orbit simulation including the gravitational acceleration caused by the Earth's gravitational field is necessary. The calculation of the gravitational influence and the integration of the equation of motion are realized within the framework of the generic simulation tool High Performance Satellite Dynamics Simulator (HPS) [9]. The HPS is a MATLAB/Simulink library which is developed at ZARM in cooperation with the DLR Institute of Space Systems, Bremen. The main focus of HPS is the propagation of satellite orbits and the computation of the satellite's orientation, depending on specific initial conditions and the space environment. Furthermore, the coupled motion of up to eight on-board test masses (arranged pairwise in up to four accelerometers) can be computed in six degrees of freedom.

Coupling effects between the satellite and the test masses as well as among the test masses themselves are included in the implemented differential equation systems of each considered body. In the following the satellite's equations of motion are shown exemplarily. The satellite motion is given by (1):

$$
\overrightarrow{\dot{r}}_{i, b}^{i}=\vec{g}_{i, b}^{i}\left(\vec{r}_{i, b}^{i}\right)+\frac{\vec{F}_{\text {control }}^{i}+\vec{F}_{\text {dist }}^{i}+\vec{F}_{\text {coupl,sat }}^{i}}{m_{\text {sat }}}
$$

Here (i) $m_{\text {sat }}$ is the mass of the satellite, (ii) $\overrightarrow{\dot{r}}_{i, b}^{i}$ is the acceleration of the satellite relative to the ECI frame, (iii) $\vec{g}_{i, b}^{i}\left(\vec{r}_{i, b}^{i}\right)$ is the gravitational acceleration, (iv) $\vec{F}_{\text {control }}^{i}$ is the control force, (v) $\vec{F}_{\text {dist }}^{i}$ is the sum of all disturbance forces acting on the satellite, and (vi) $\vec{F}_{\text {coupl,sat }}^{i}$ is the force due to the coupling between the satellite and all considered test masses. The superscript $i$ indicates that all components of (1) are given in ECI coordinates. 
The satellite's rotation and the satellite's attitude motion are computed by using (2) and (3):

$$
\begin{aligned}
& \vec{\omega}_{i, b}^{b} \\
& \quad=\left(\mathbf{I}_{b}^{b}\right)^{-1}\left[\vec{T}_{\text {control }}^{b}+\vec{T}_{\text {dist }}^{b}+\vec{T}_{\text {coupl,sat }}^{b}-\vec{\omega}_{i, b}^{b} \times\left(\mathbf{I}_{b}^{b} \vec{\omega}_{i, b}^{b}\right)\right], \\
& \dot{\mathbf{q}}_{i}^{b}=\frac{1}{2} \vec{\omega}_{i, b}^{b} \odot \mathbf{q}_{i}^{b} .
\end{aligned}
$$

Here (i) $\omega_{i, b}^{b}$ is the angular velocity of the satellite relative to the ECI frame, (ii) $\mathbf{I}_{b}^{b}$ is the moment of inertia matrix of the satellite, (iii) $\vec{T}_{\text {control }}^{b}$ is the sum of the control torques applied for attitude control, (iv) $\vec{T}_{\text {dist }}^{b}$ are the disturbance torques acting on the satellite, (v) $\vec{T}_{\text {coupl,sat }}^{b}$ are the torques generated from the satellite-test mass coupling, (vi) $\vec{\omega}_{i, b}^{b}$ is the quaternion representation of $\vec{\omega}_{i, b}^{b}$, and (vii) $\mathbf{q}_{i}^{b}$ represents the Euler symmetric parameters. Here the superscript $b$ indicates a description of the equations' components in the satellite body fixed frame.

It is obvious that the satellite's motion is affected by the acceleration due to the Earth's gravitational field which cannot be considered to be spherically symmetric. This is due to the nonuniform mass distribution of the Earth and it results in (i) perturbations of the pure Kepler orbit and in (ii) perturbations of the satellite's attitude. But apart from this, for a complete orbit and attitude propagation simulation, one has to take into account nongravitational effects acting on the satellite, too. They force it to go astray from its purely gravitational orbit and induce undesired rotations. For many missions, one of the most prominent effects of these NGDs is the SRP which will be discussed in detail in the next sections.

\section{SRP Model}

The disturbance forces and torques due to SRP originate from the interaction of the satellite's surface with the photons emitted by the sun. It is assumed that each photon that hits the satellite is either absorbed or reflected in a specular or diffuse way, thus effectively changing the momentum of the satellite. As a consequence, the resulting force acting on an elemental area $d A$ can be expressed as the sum of three individual contributions [10]:

$$
\begin{aligned}
d \vec{F}_{\alpha} & =-P_{\text {SRP }} \alpha \cos (\theta) \vec{e}_{\text {Sun }} d A, \\
d \vec{F}_{\gamma_{S}} & =-2 P_{\text {SRP }} \gamma_{S} \cos ^{2}(\theta) \vec{e}_{N} d A, \\
d \vec{F}_{\gamma_{D}} & =P_{\text {SRP }} \gamma_{D}\left(-\frac{2}{3} \vec{e}_{N}-\vec{e}_{\text {Sun }}\right) \cos (\theta) d A,
\end{aligned}
$$

where (i) $P_{\text {SRP }}$ is the SRP, (ii) $d A$ is the elemental area, (iii) $\vec{e}_{\text {Sun }}$ and $\vec{e}_{N}$ are the unit vector in Sun direction and the unit vector normal on the elemental area $d A$, respectively, and (iv) $\theta$ is the angle between $\vec{e}_{\text {Sun }}$ and $\vec{e}_{N}$. Finally, (v) $\alpha, \gamma_{S}$, and $\gamma_{D}$ are the coefficients of absorption, of specular reflection, and of diffuse reflection.
With $\alpha+\gamma_{S}+\gamma_{D}=1$ (assuming a nontransparent material) the force due to SRP can be derived as follows:

$$
\begin{aligned}
& \int d \vec{F}_{\text {total }}=-P_{\text {SRP }} \int\left[\left(1-\gamma_{S}\right) \vec{e}_{\text {Sun }}\right. \\
& \left.+2\left(\gamma_{S} \cos (\theta)+\frac{1}{3} \gamma_{D}\right) \vec{e}_{N}\right] \cos (\theta) d A
\end{aligned}
$$

Hence, the computation of $\vec{F}_{\text {total }}$ requires the modelling of

(i) the satellite orbit because the magnitude of $P_{\text {SRP }}$ depends on the distance to the Sun,

(ii) the satellite attitude in order to derive the correct incident angle $\theta$ between $\vec{e}_{N}$ and $\vec{e}_{\text {Sun }}$,

(iii) the satellite geometry for defining appropriate values of $\gamma_{S}, \gamma_{D}, \vec{e}_{N}$, and $A$.

The propagation of the satellite orbit and its orientation is one of the basic tasks within the simulation software HPS and can be applied to any Earth orbiting satellite mission. Since most NGDs such as the SRP are surface-based effects, the propagation needs an input model for the satellite geometry specific to the actual mission. Due to the variations in satellite components, general dimensions, and external materials it is not possible to find a suitable standard model that can be used in a flexible way with respect to the variety of spacecraft geometries. This is one of the main setbacks of standard approaches such as the Cannonball or the Wing-Box model.

Instead of using a simplistic approach, where $\vec{F}_{\text {total }}$ is calculated with respect to (i) an effective projected satellite surface area and to (ii) averaged optical surface properties, the focus of the HPS SRP interface lies on the capturing of the influence of details of the satellite geometry and the involved material parameters of each component of the satellite. In order to realize this, the HPS SRP approach is divided into two main steps. Before the actual SRP is calculated, the satellite's surface is discretized in small elements $A_{i}$ and the different optical properties are assigned to the corresponding elements. For a complex geometry this is realized by means of a finite element (FE) preprocessor, where the meshes of the external surfaces are exported together with their respective optical property definitions. Subsequently, the HPS algorithm for SRP computation evaluates (6) which is the discrete form of (5) for each element that is illuminated by the Sun for the chosen vector $\vec{e}_{\text {Sun }}$ :

$$
\begin{aligned}
\vec{F}_{i} & =-P_{\text {SRP }}\left[\left(1-\gamma_{S i}\right) \vec{e}_{\text {Sun }}\right. \\
& \left.+2\left(\gamma_{S i} \cos \left(\theta_{i}\right)+\frac{1}{3} \gamma_{D i}\right) \vec{e}_{N i}\right] \cos \left(\theta_{i}\right) A_{i}
\end{aligned}
$$

The overall force is then derived by computing the sum over all elements:

$$
\vec{F}_{\mathrm{SRP}}=\sum_{i} \vec{F}_{i}
$$

By means of geometric criteria (see [11] for details), the algorithm determines automatically if an element is lit by the 
Sun and considers shadowing by other parts of the satellite as well. In combination with an eclipse model, the global SRP acting on the satellite is calculated with respect to a realistic illumination scenario.

In order to speed up the simulation process, resulting SRP magnitude and directions can be derived in a normalized form. Here a lookup table can be derived where parameters of the stored SRP values are the solar elevation and azimuth consequently defining the current sun angle. When the lookup table is computed in preprocessing, the results can be used to determine the dynamical evolution of the SRP during flight within an HPS simulation. For this, the normalized SRP values are converted to the actual SRP with respect to the current solar distance and orientation of the satellite as well as the eclipse condition.

\section{Parameter Analysis}

In order to review the systematics of the SRP force model discussed here, a parameter analysis is performed. The implications of changes of the relevant input parameters such as orbital elements and geometrical and technical features with respect to the overall magnitude of the resulting disturbance force due to SRP are discussed in the following.

4.1. Solar Radiation Pressure. Since the magnitude of the incident solar radiation does depend not only on the orbit of the satellite around the Earth but rather on the Earth's orbit around the Sun, too, it is sensible to analyse the influence of the implications of the central body orbit during the year. The annual variation of the strength of $P_{\mathrm{SRP}}$ is depicted in Figure 1. Exemplarily, the resulting SRP in $\mathrm{Nm}^{-2}$ for the CHAMP mission orbit (see Table 1 for details) is presented.

Furthermore, the resulting SRP values for the Low Earth Orbit (LEO) missions CHAMP and MICROSCOPE (sunsynchronous orbit (SSO)), the SRP detected for the orbit of the geostationary (GEO) mission Meteosat, and the disturbing radiation pressure for the GALILEO satellites are given in Figure 2 for the timeframe of a single day.

The detected strength of the SRP varies on large time scales due to the change of the distance between the Earth and the Sun over the time period of one year (see Figure 1). The variations of the SRP on smaller time scales (see Figure 2) are a consequence of the satellites' motion around the Earth, resulting in additional distance variations with respect to the Sun. Figure 1 shows that in case of CHAMP the SRP changes about $6 \%$ during a half-year period (from winter to summer). The magnitude of the small scale variations depends considerably on the type of the satellite's orbit, that is, LEO, GEO, and so forth. This is demonstrated in Figure 2.

Another effect is the variability of the SRP due to changing solar activity. The main variation of the intensity of solar radiation shows a period of eleven years. The corresponding variation of the amplitude of the solar constant, which is the total solar irradiance (TSI) at a fixed distance of one AU, is only $0.1-0.2$ percent [12]. Due to the relative small variation compared to the variation induced by the elliptic Earth orbit this effect is negligible and will not be taken into account in the following.

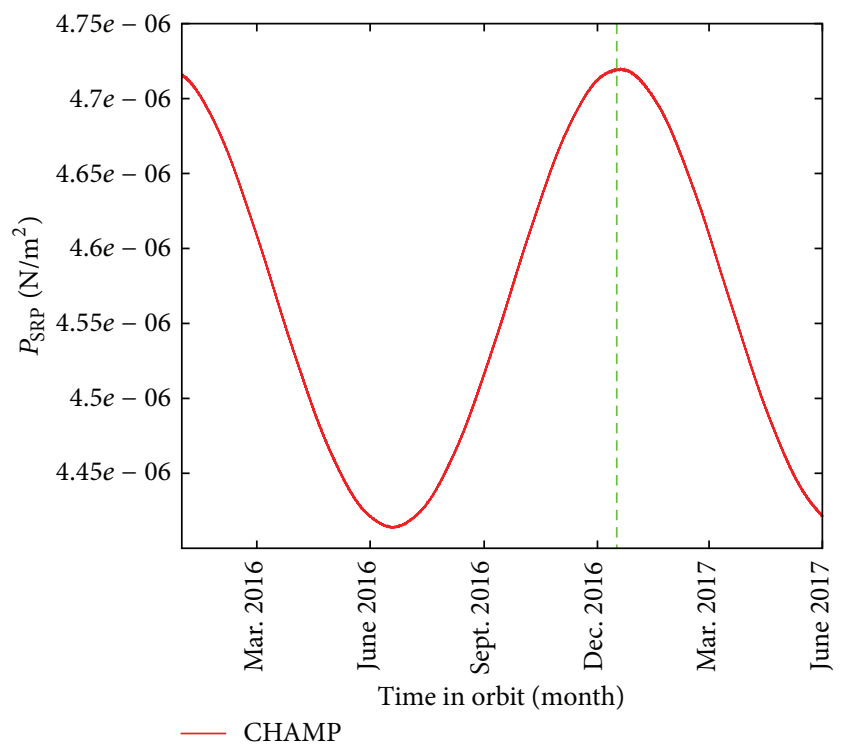

FIGURE 1: Annual variation of the SRP for satellites orbiting the Earth. Exemplarily, the orbit of CHAMP is depicted.

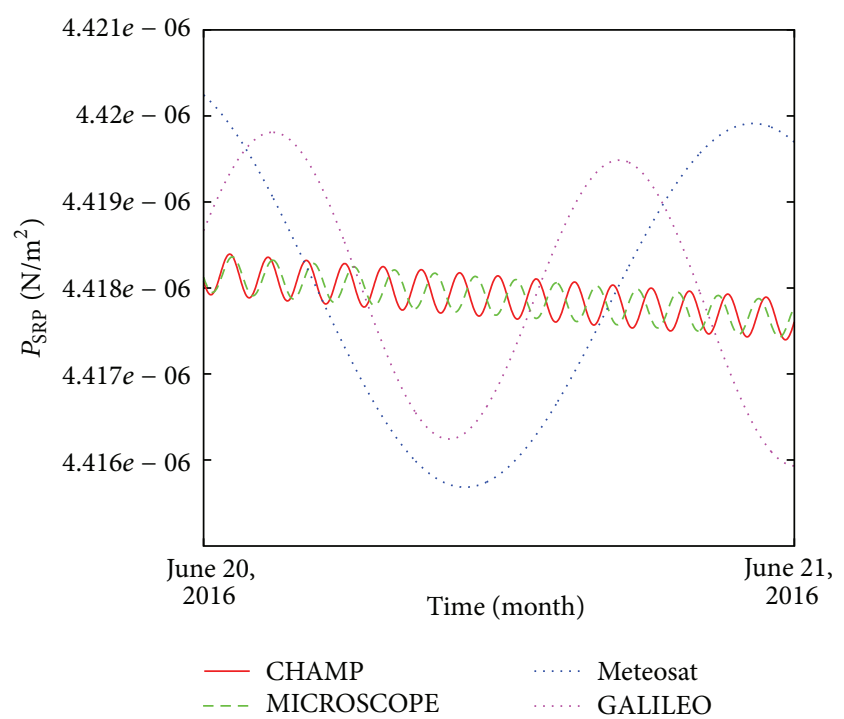

FIGURE 2: Resulting SRP for selected satellite missions. The variation over one day is shown.

The choice of example missions is based on Table 1 which provides an overview about the distribution of operating satellites on the different mission classes. As each category is linked with a typical altitude, the above-named conclusions can be interpreted as a general survey of the evolution of SRP for a broad range of satellite missions.

4.2. Geometry Models. In contrast to the general analysis of $P_{\mathrm{SRP}}$, an investigation of the influence of satellite attitude and design, that is, its geometry and the surface materials, requires higher effort and will be carried out exemplarily for the MICROSCOPE mission. 
TABLE 1: Overview of orbit classes including typical orbit parameters and mission examples.

\begin{tabular}{|c|c|c|c|c|c|c|}
\hline Orbit category & Percentage & Inclination $i\left[^{\circ}\right]$ & Altitude $[\mathrm{km}]$ & Semimajor axis $[\mathrm{km}]$ & Mission name & Operation \\
\hline LEO & $41 \%$ & & & & & \\
\hline \multirow{2}{*}{ Examples: } & & $88.940-89.060$ & $485-500$ & $6870-6770$ & GRACE & Gravity field recovery \\
\hline & & 87.180 & 454 & 6823 & CHAMP & Earth observation \\
\hline GEO & $8 \%$ & & & & & \\
\hline \multirow{4}{*}{ Examples: } & & 0.028 & 36000 & 42162 & Meteosat & Meteorology \\
\hline & & 0.180 & 35780 & 42160 & GOES & Meteorology \\
\hline & & 0.020 & 42161 & 42165 & Arsat-1 & Communication \\
\hline & & 0.040 & 35796 & 42165 & Ciel-2 & Direct-broadcast \\
\hline GTO \& HEO & $12 \%$ & $\begin{array}{c}7.000 \\
\text { (Kourou) }\end{array}$ & $\begin{array}{c}250 \text { (perigee) } \\
36000 \text { (apogee) }\end{array}$ & 24582 & & Transfer \\
\hline SSO & $31 \%$ & & & & & \\
\hline \multirow{4}{*}{ Examples: } & & 98.248 & 700 & 7078 & MICROSCOPE & Science \\
\hline & & 98.600 & $780-800$ & 7145 & Envisat & Earth observation \\
\hline & & 98.390 & 714 & 7084 & SwissCube-1 & Science \\
\hline & & 97.469 & 510 & 6885 & Belka 2 & Earth observation \\
\hline MEO & $7 \%$ & & & & & \\
\hline \multirow{3}{*}{ Examples: } & & 56.000 & 23222 & 29601 & GALILEO & Navigation \\
\hline & & 64.800 & 19100 & 25510 & GLONASS & Navigation \\
\hline & & 55.000 & 20180 & 26580 & GPS & Navigation \\
\hline Molniya & & 63.400 & $\begin{array}{l}7378 \text { (perigee) } \\
45730 \text { (apogee) }\end{array}$ & 26554 & Molniya satellites & Communication \\
\hline
\end{tabular}

MICROSCOPE will be operated on a sun-synchronous orbit at an altitude of $700 \mathrm{~km}$ and an inclination of $98.248^{\circ}$. In order to provide a stable thermal environment for the payload and to minimize eclipse phases MICROSCOPE will be injected in an orbit with 6:00 hrs or 18:00 hrs local solar time at ascending node. Figure 3 illustrates the attitude of MICROSCOPE with respect to its orbital plane.

As stated above, a usual simplification of the satellite's geometry involves the definition of a reference area with mean values for the optical properties. In contrast the HPS concept utilises FE models which demand a certain effort during construction. Between these approaches a range of other geometry models is of common usage. For example, due to its symmetry a sphere may be used as very simple model for the geometry of a satellite. This so-called Cannonball model [13] results in SRP forces completely independent of the attitude if all surfaces share the same optical properties.

In reality, satellites possess a more or less complex geometry. The total value of the force can strongly depend on the incident angle even in the case of a homogeneous distribution of optical parameters on the external surfaces. In particular, flat components like solar panels contribute to this dependency. For this reason so-called Wing-Box models are used. They offer the possibility to introduce different optical properties, generally for the satellite body and the solar panels [14].

In addition to the complex FE model we generated different geometry models to demonstrate the impact of geometric complexity on the resulting SRP effects including the most simple approach (disk), a simple box, and a WingBox model. They are depicted in the upper row of Figure 4. In order to get the best comparability we set the same projected surface area for each model (with $\theta=90^{\circ}$ and $\varphi=0^{\circ}$, corresponding to the MICROSCOPE solar panel side). In addition, a spherical geometry model was chosen to provide a global comparative value for this analysis. The model is not shown in Figure 4 for reasons of brevity. In the lower row of Figure 4, the values of the projected surface areas for the different geometry models are depicted as a function of the incident sunray described in polar coordinates (small picture in Figure 3). In each case the comparative value of the spherical geometry model appears as constant surface area independent of $\varphi$ and $\theta$. Usually, the disk model is only applicable for vertical incident sunlight. Therefore, it is not expected to be a good choice for MICROSCOPE, as its solar panels will not be exposed to perpendicular solar irradiation most of the time. This results from the fact that the satellite's $x$-axis will be aligned with the orbit normal and not with the vector to the Sun. During the year the incident angle $\theta$ varies in the range of about $30^{\circ}$ which results from the combination of inclination and obliquity of the ecliptic. The simple box, the Wing-Box, and the FE models show characteristic results that represent the symmetries of each of the models. Obviously the simple box model results in large deviations from the FE model, especially for angles $\theta$ far from $90^{\circ}$ because it does not take into account the geometry of the solar panels and the corresponding correct contribution to the total area for these angles. In general, the Wing-Box model gives a good representation of the projected area, but the distribution for the FE model is much smoother. Furthermore, the definition of the reference projected area yields an overestimation of 


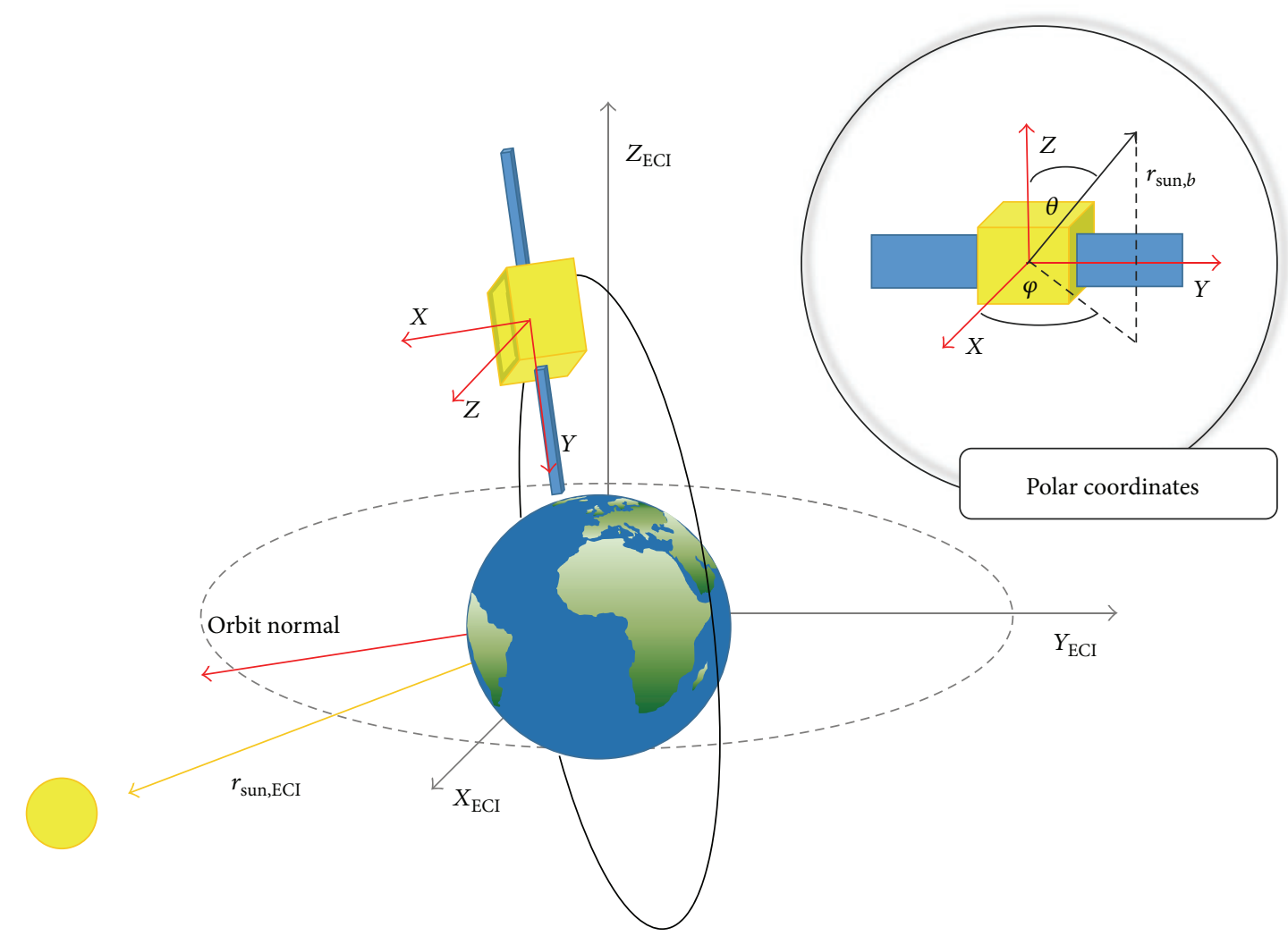

FIGURE 3: Illustration of MICROSCOPE orbit with respect to Earth-centred inertial coordinates (ECI). Small picture: definition of vector to the Sun in polar coordinates $\theta$ and $\varphi$.

the projected area for $\theta \neq 90^{\circ}$ for both the simple box and the Wing-Box models. In summary the FE model produces the most accurate results for the projected area.

Another reason for using at least simple box models is the fact that different optical properties can be assigned to the single satellite surface cells. Figure 5 shows the FE model of MICROSCOPE in which the different materials are represented. Each color corresponds to specific values of $\gamma_{\mathrm{S}}$ and $\gamma_{D}$.

The influence of the optical properties is demonstrated in Figure 6. Here the absolute value of $\vec{F}_{\mathrm{SRP}}$ is depicted as function of $\varphi$ and $\theta$ for a constant value of $P_{\text {SRP. }}$. In contrast to Figure $4(\mathrm{~d})$ there is a significant difference between the peak at $\theta=90^{\circ}$ and $\varphi=0^{\circ}$ corresponding to the solar panel side and the opposite side $\left(\theta=90^{\circ}, \varphi=180^{\circ}\right)$ although the projected area is nearly the same. Looking at the material distribution, the result is not surprising. The back side of the solar panels is covered with White Paint. Consequently the corresponding surface cells have higher reflection coefficients and contribute stronger to the absolute values of $\vec{F}_{\mathrm{SRP}}$ compared to those on the front side. Overall, the difference between both sides for perpendicular solar irradiation amounts to approximately $13 \%$.

When detailed surface models are used, the quality of the obtained force considerably depends on the chosen mesh. On the one hand, geometrical features such as spherical bodies can only be implemented realistically with a considerably small meshed surface grid. The same effect shows in the illumination condition calculations where the shape of the shadow improves with a higher number of elements. On the other hand, computation time considerably increases with a finer mesh. Here the computation of shadowing is the dominant effect. Every surface element has to be checked for shadowing considering its orientation and position with respect to each other surface element included in the model. Besides the obvious quadratic increase of the number of individual computation steps, also the size of the data matrices needed to store the shadowing information increases at the same rate. As a consequence, a trade-off between computational resources available and accuracy demands has to be made. Keeping in mind that the actual illumination condition has to be recalculated for different orientations of the satellite to the Sun, the surface mesh has to be chosen such that acceptable computation times can be realized while the quality of the illumination implementation is not compromised. A suitable method to obtain a good tradeoff is to calculate the projected illuminated area surface at a steep illumination angle (consequently causing long shadows) for different mesh qualities. Figure 7 shows the resulting calculated illuminated surface area for a different number of surface elements. The mean element edge length $l_{e}$ is used as a mesh criterium and ranges from $50 \mathrm{~cm}$ to $2 \mathrm{~cm}$, resembling the range of the displayed model number $n$ (with $n=1, \ldots, 25$ ), where $l_{e}=1 /(n \cdot 2)$. Keeping in mind 

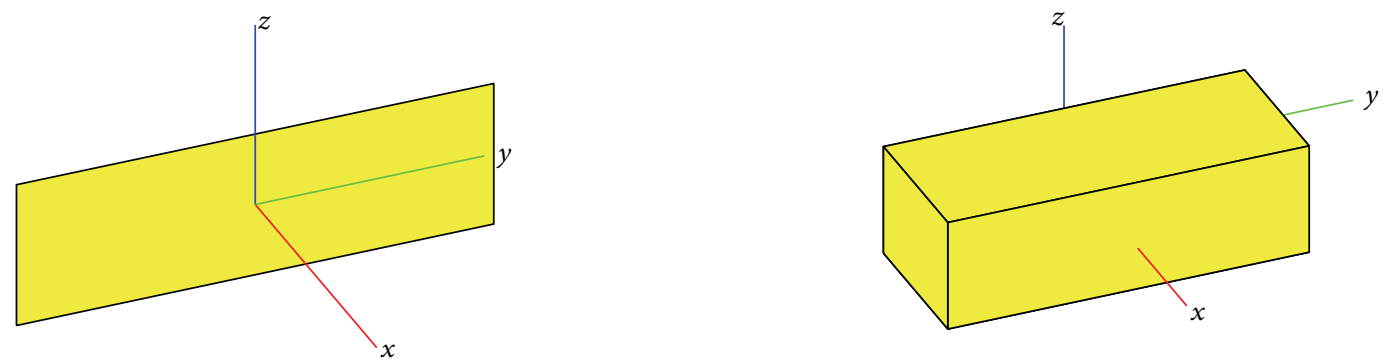

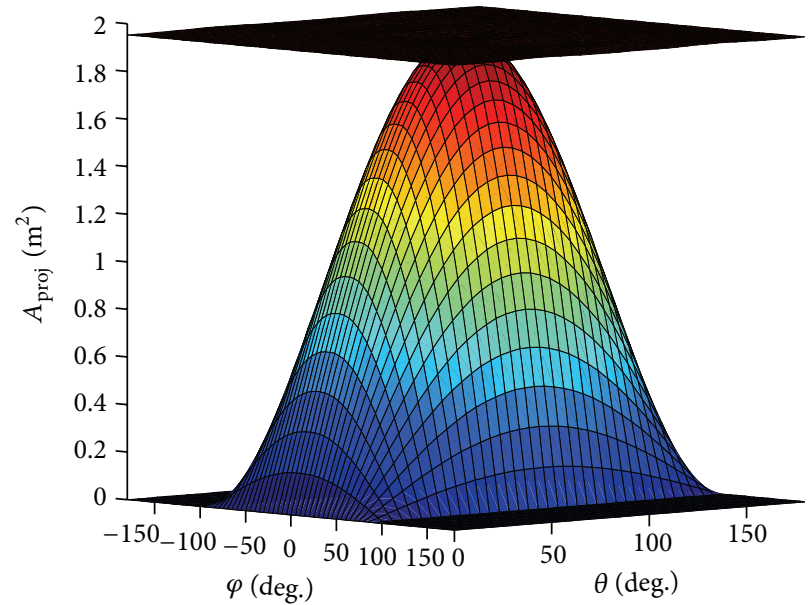

(a) Disk

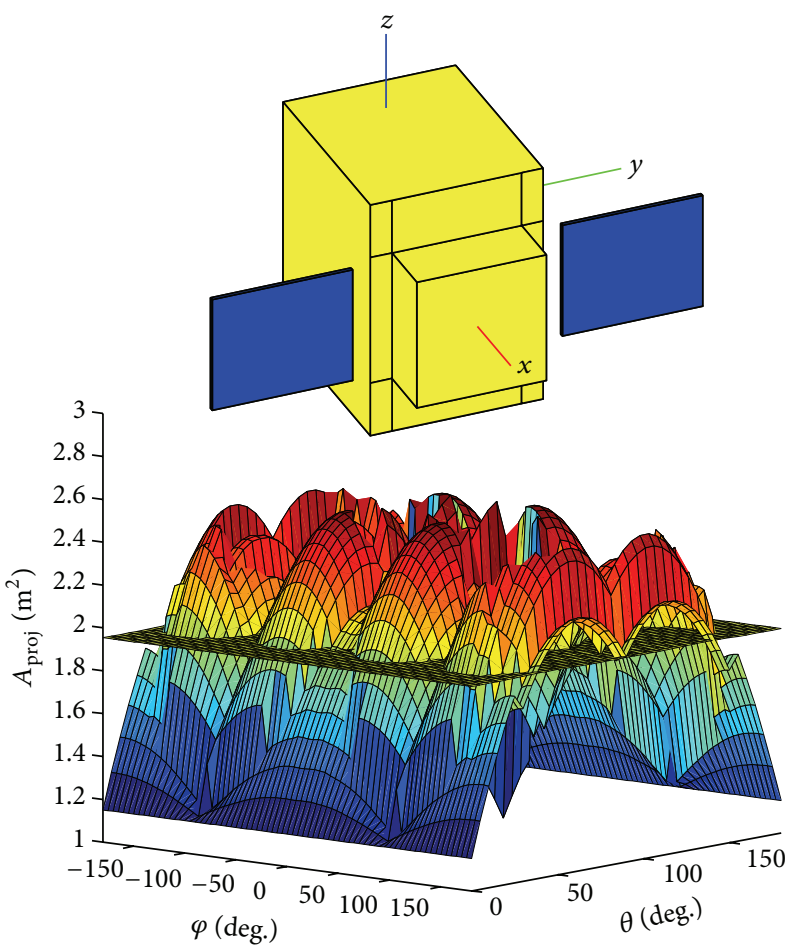

(c) Wing-Box

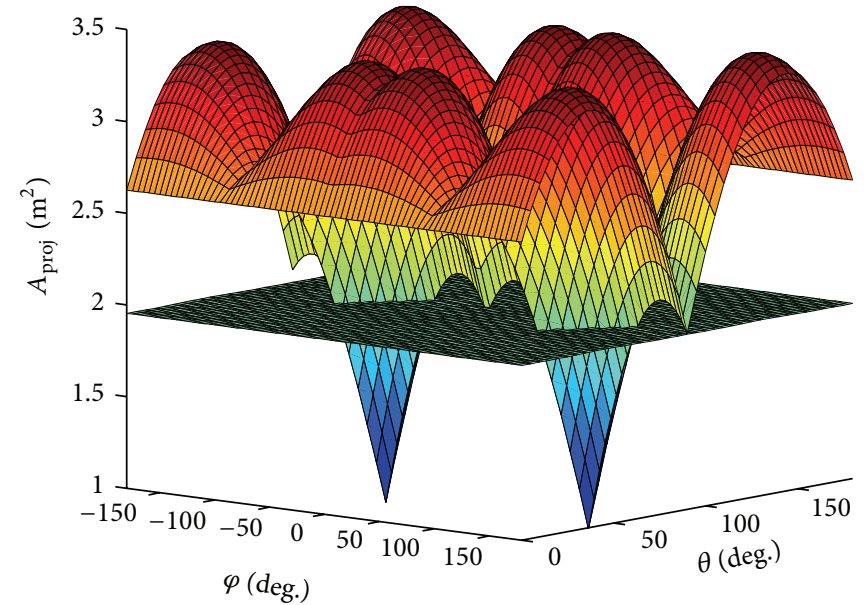

(b) Simple box
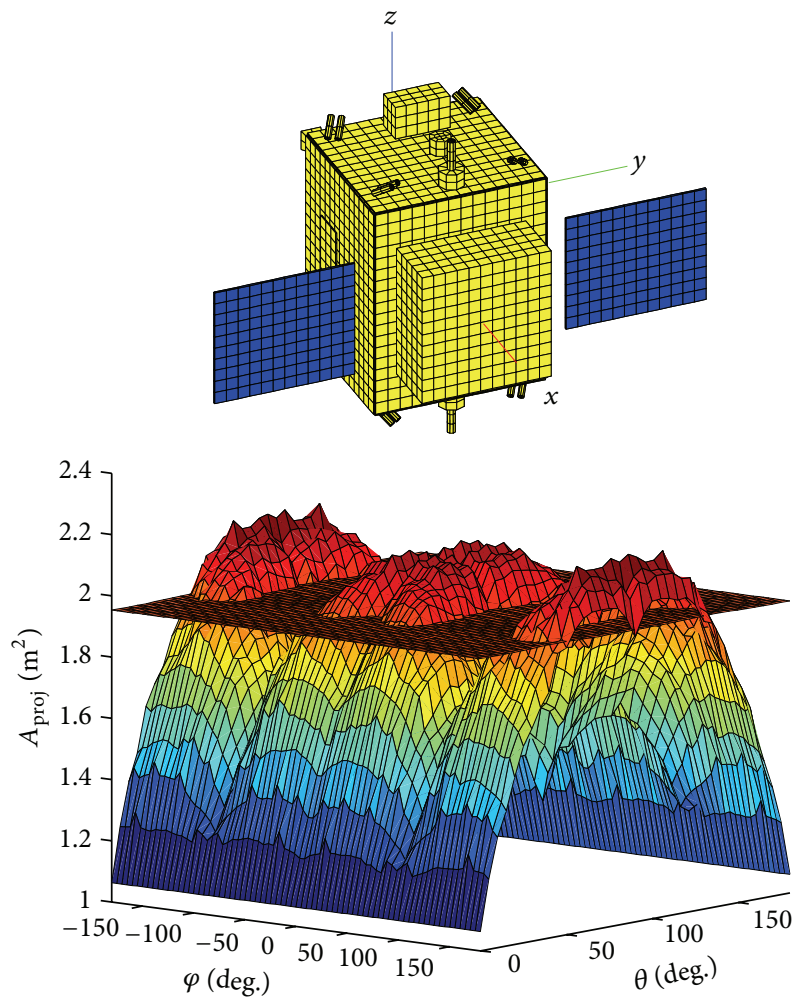

(d) FEM

FIGURE 4: Upper part (a, b, c, and d): different geometry models for the SRP computation with same projected area in $x$-direction. Lower part $(\mathrm{a}, \mathrm{b}, \mathrm{c}$, and $\mathrm{d})$ : projected area $\left(A_{\text {proj }}=\cos (\theta) A\right)$ as function of incident sunray in polar coordinates $\varphi$ and $\theta$ for the corresponding models and the sphere model (constant level). 


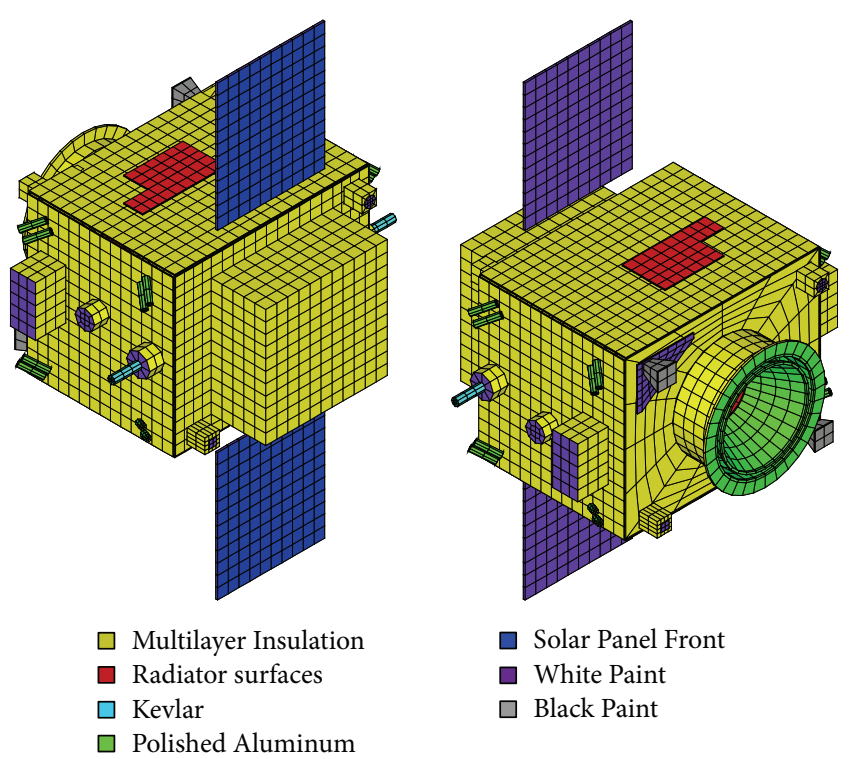

FIGURE 5: Definition of surface materials in the FE model of MICROSCOPE.

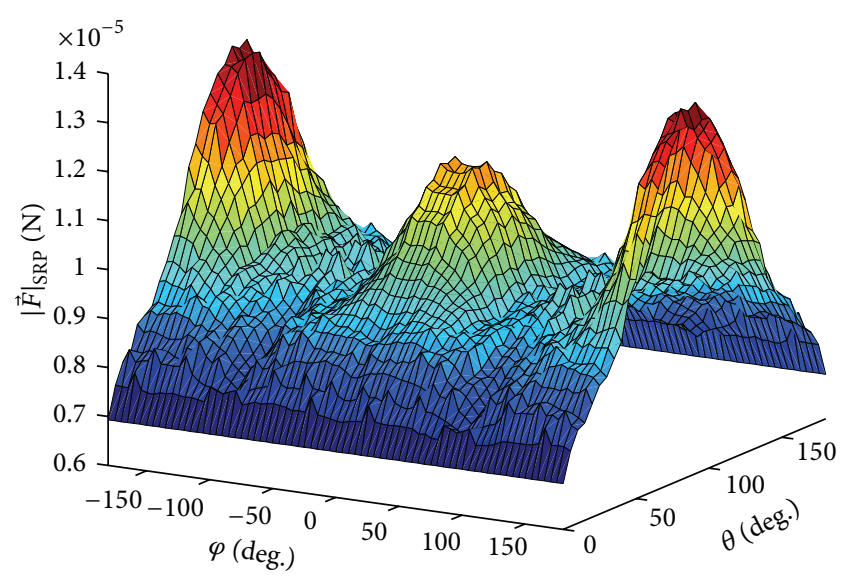

FIGURE 6: $|\vec{F}|_{\text {SRP }}$ as function of incident sunray in polar coordinates $\varphi$ and $\theta$ with a constant value of $P$ for the FE model of MICROSCOPE.

the bus size of MICROSCOPE (e.g., $+X$ bus side of about $1.1 \mathrm{~m} \times 0.8 \mathrm{~m})$, the first model $(n=1)$ translates to four elements on the $+X$ bus face, while the last model $(n=25)$ translates to more than two thousand elements on the same surface. A mean value of the obtained area is displayed as a grey dashed line. As can be seen, the solution converges close to the mean value for a fine mesh. However, reducing the element edge size does not directly lead to a better surface area result. The actual element size depends (i) on the region boundary lines, (ii) on the meshing sequences, and (iii) on the free parameters specified by the meshing tool (ANSYS classic preprocessor). The exact values for these parameters may vary for different element edge lengths. When processed for different illumination angles, a mean element edge size $l_{e}=6.25 \mathrm{~cm}$ shows projected areas close to the arithmetic mean. Additionally, for this chosen value of $l_{e}$ the processing

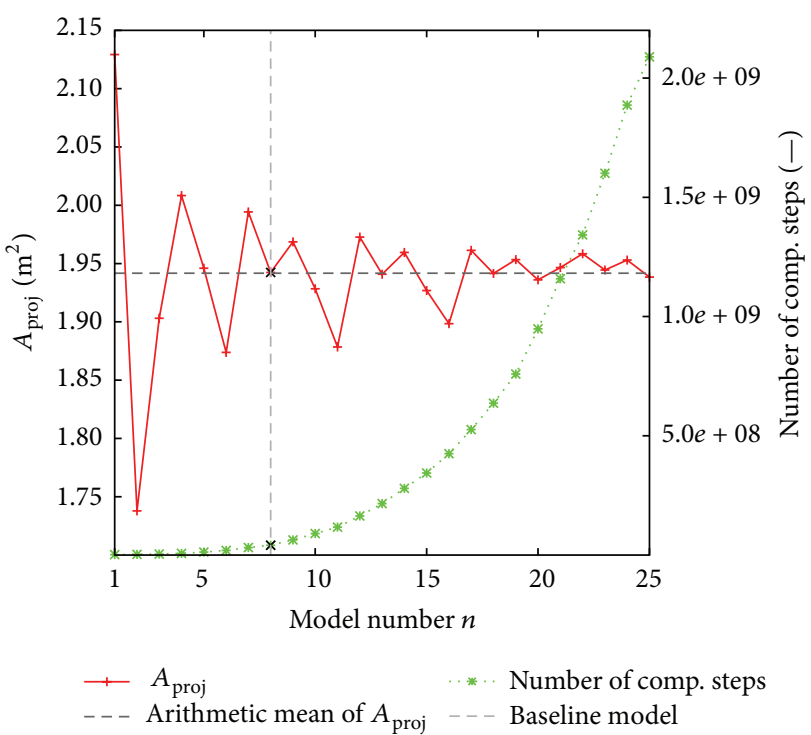

FIGURE 7: Investigation of convergence for the projected area of the FE model. The arithmetic mean of the projected area is represented by the dashed gray line. Additionally, the appropriate numbers of computational steps are shown. The black asterisk (together with the light gray dashed line) marks the model of choice.

time for a complete assessment of all illumination conditions with a $5^{\circ}$ resolution in elevation and azimuth angle is in the range of $30 \mathrm{~min}$ for a conventional desktop PC, which is still acceptable. Consequently, the mesh resulting from $l_{e}=$ $6.25 \mathrm{~cm}$ is the baseline for all further calculations in this work. However, since the optimal configuration highly depends on the actual satellite shape and the positions of its components an optimal mesh has to be assessed for each new satellite that has to be processed.

4.3. Combined Effect of SRP and Geometry Models. As seen above both the geometrical dependency of the SRP force and the dynamical behaviour of $P_{\mathrm{SRP}}$ determine the resulting total SRP force acting on the satellite. Consequently, we investigate the behaviour of the SRP force acting on MICROSCOPE with both effects included in the modelling approach. In Figure 8 each line in both pictures represents the absolute value of $\vec{F}_{\text {SRP }}$ for a specific $\vec{e}_{\text {Sun }}$ : that is, it is assumed that the satellite's orientation is fixed with respect to the Sun over one year. In the top picture the outcome for normal incident sunlight for each satellite side is depicted. In order to compare more realistic illumination conditions, we considered deviations from the normal vector of the solar panel side of $15^{\circ}$ and $30^{\circ}$, respectively, which is depicted at the bottom of Figure 8.

This resembles the range that is expected for MICROSCOPE. Naturally, all lines show the same characteristics due to the variation of $P_{\mathrm{SRP}}$ over the year which yield a maximum difference in magnitude of about $7 \%$. However, the influence of the satellite's attitude might result in larger differences, for example, $13 \%$ for $\pm X$ as seen above. For the MICROSCOPE case differences of roughly $1 \%$ are obtained for deviations from the normal axis of $+X$ of $15^{\circ}$ and $9 \%$ for deviations of $30^{\circ}$. 


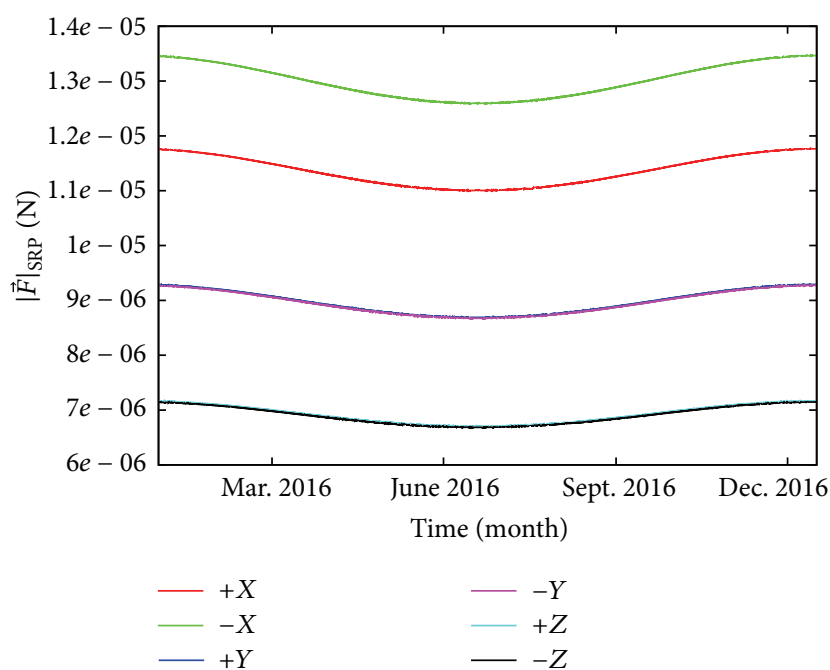

(a)

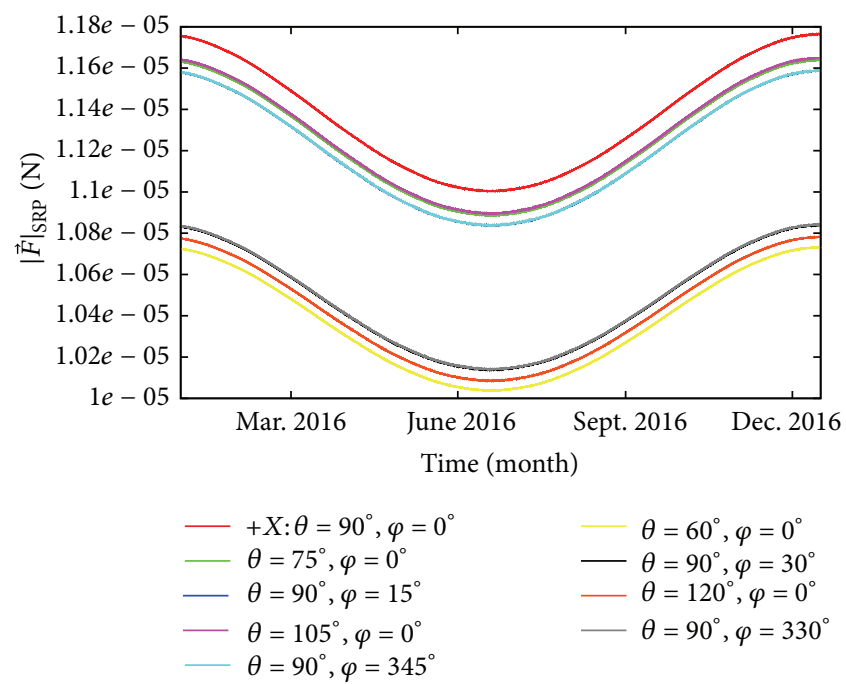

(b)

FIGURE 8: Absolute value of disturbance force due to SRP for different illumination conditions depicted over one year. (a) Perpendicular solar irradiation of each satellite side. (b) Illumination conditions estimated for MICROSCOPE.

Finally, a simulation of the MICROSCOPE orbit for a simulation time of one year was carried out with all five geometry models. Figure 9 shows the resulting evolution of $|\vec{F}|_{\text {SRP }}$.

Only for the sphere model, the effect of the changing distance between Sun and Earth becomes visible. For all other models, the variation of the incident sunlight is the dominating effect. For the FE model, the force differs about $16 \%$ from the maximum in winter to the minimum in summer. Besides Figure 9 shows an unexpected result: the disk model performs better for the MICROSCOPE scenario which is in contrast to the assumption that a Wing-Box model will resemble the results of a FE model best (according to the projected area in Figure 4).

Furthermore, there are steep changes in the evolution of the resulting SRP force that only appear for the WingBox model. Figure 10 reveals the problem that occurs for this modelling approach. Here the calculated illumination conditions for both the Wing-Box and the FE model are depicted for two different dates. The first one is chosen at the end of April 2016 right before the steep decrease (upper row) and the second one only a few days later, directly after this strong decrease (lower row). The chosen scenarios are marked with black asterisks in Figure 9. For the FE model the shaded area (red elements) changes little due to the modified incoming sunlight. But for the Wing-Box model, the side panel changes from fully sunlit to completely shaded and therefore does not contribute to the force anymore. Such effects cannot appear for the disk model which yields a smoother evolution of the force. This outcome emphasizes that each scenario has to be investigated individually in order to obtain the best result.

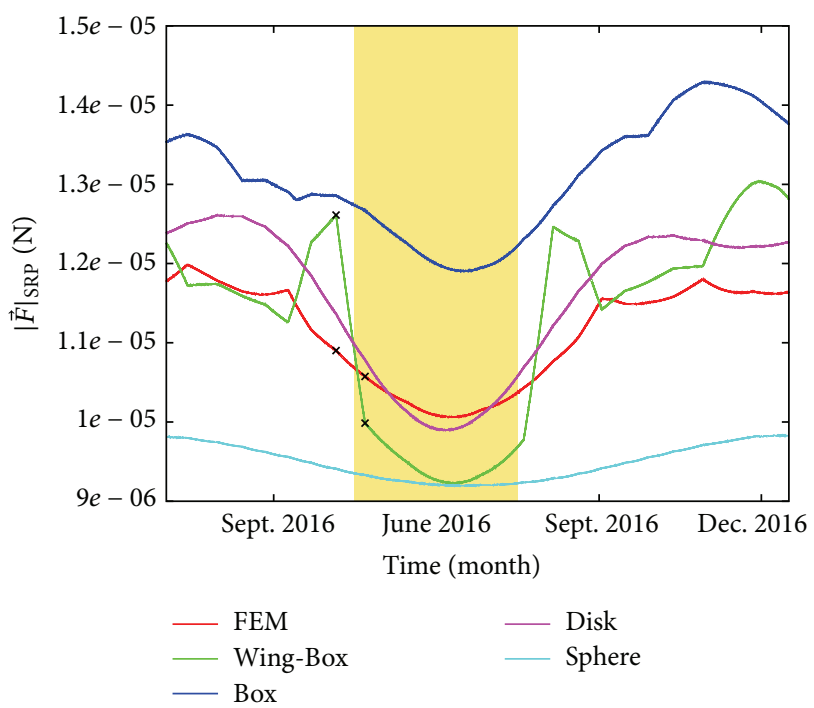

FIGURE 9: Resulting disturbance force due to SRP for different geometry models over one year. The yellow bar marks the time of eclipse.

4.4. Degradation Influence. In the sections above, it was assumed that the optical properties do not change during the mission. In a more realistic approach accurate SRP models also have to allow for surface degradation effects that occur when external surfaces are exposed to the space environment. Material degradation may effectively change the resulting SRP force magnitude and orientation. Different materials show different sensitivity and different degradation 


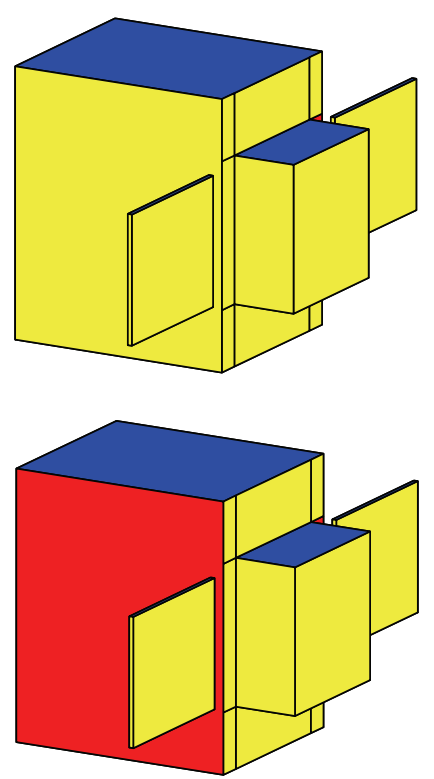

(a)
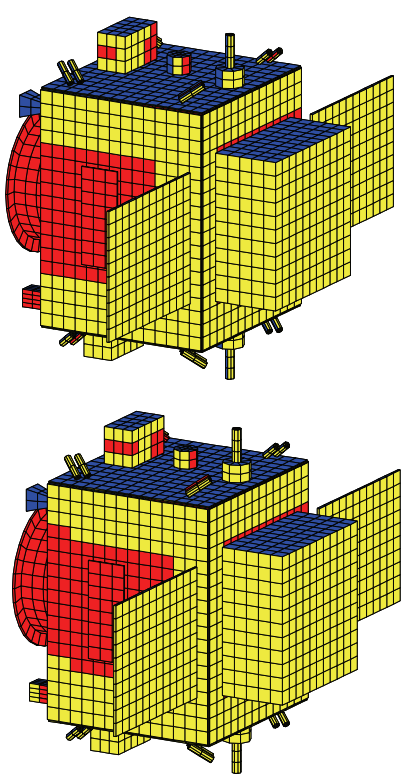

(b)

FIGURE 10: Illumination conditions of Wing-Box model (a) and FE model (b) for different dates of the simulated MICROSCOPE scenario. Upper row: end of April 2016, lower row: a few days later in May 2016. Yellow elements are in full sunlight, blue elements are not exposed to the Sun, and red elements are shadowed by other parts of the satellite.

behaviour with respect to atomic oxygen, space debris, radiation, and thermal cycles [15]. However, for most materials used in space, the mean coefficient of absorptivity (with respect to the solar spectrum) will increase over time, while the mean coefficient of emissivity will not show a drastic change.

In order to test the influence of a degradation of the optical properties of external surfaces to the resulting SRP, a variation of solar absorptivity over mission lifetime is considered. Again MICROSCOPE is used as test case. In order to define a model for the degradation rate, a logarithmic evolution of the absorptivity is considered. Assuming that surface degradation leads to a microscopic cratering, effectively the increase of absorptivity will depend on the increase of surface area resulting from the roughened surface. As a consequence, the rate of change in $\alpha$ will be high during the first months of mission and decrease over mission time. A suitable model for this behaviour is a reciprocal dependency of the time derivative of the mean coefficient of absorptivity $\alpha$ on the time $t$ :

$$
\frac{d \alpha}{d t}=p \cdot \frac{1}{t+1}
$$

leading to

$$
\alpha=\alpha_{\mathrm{BOL}}+p \cdot \ln (t+1)
$$

with

$$
p=\frac{\alpha_{\mathrm{EOL}}-\alpha_{\mathrm{BOL}}}{\ln (T+1)},
$$

where $T$ is the total mission lifetime and $p$ is the degradation rate scaling factor. The begin-of-life (BOL) and end-of-life
TABLE 2: Considered BOL and EOL values for $\alpha, \varepsilon, \gamma_{S}$, and $\gamma_{D}$. MLI: Multilayer Insulation, SPF: Solar Panel Front, WP: White Paint, KV: Kevlar, PA: Polished Aluminum, RAD: Radiator surface, and BP: Black Paint.

\begin{tabular}{lccccccc}
\hline Component & MLI & SPF & WP & KV & PA & RAD & BP \\
\hline$\alpha_{\text {BOL }}$ & 0.42 & 0.92 & 0.24 & 0.73 & 0.12 & 0.08 & 0.97 \\
$\alpha_{\text {EOL }}$ & 0.5 & 0.92 & 0.30 & 0.73 & 0.15 & 0.18 & 0.97 \\
$\varepsilon$ & 0.84 & 0.85 & 0.88 & 0.92 & 0.04 & 0.8 & 0.89 \\
$\gamma_{S, \text { BOL }}$ & 0.29 & 0.0727 & 0.38 & 0.2455 & 0.8 & 0.46 & 0.015 \\
$\gamma_{S, \text { EOL }}$ & 0.071 & 0.05 & 0.099 & 0.168 & 0.53 & 0.116 & 0.004 \\
$\gamma_{D, \text { BOL }}$ & 0.29 & 0.007 & 0.38 & 0.025 & 0.08 & 0.46 & 0.015 \\
$\gamma_{D, \mathrm{EOL}}$ & 0.429 & 0.030 & 0.601 & 0.102 & 0.3204 & 0.704 & 0.0257 \\
\hline
\end{tabular}

(EOL) properties as given by the MICROSCOPE mission definition [16-18] are listed in Table 2. Note that the specified values for BOL/EOL values of specular and diffuse reflectivity are modeled values since no actual data on their properties is available.

Since the total coefficient of reflectivity is given by $\gamma=\alpha$ (assuming nontransparent surfaces), (8) can also be applied for an assessment of the evolution of the coefficient of reflectivity. However, not only the total magnitude of reflection but also the ratio between specular and diffuse reflections may change. The individual evolution of $\gamma_{S}$ and $\gamma_{D}$ depends on the BOL properties of the respective surface material and the actual conditions experienced in space. Due to the lack of actual data we use a model with a qualitative approach. Since a roughening of a smooth surface causes a drop in specular reflectivity, the ratio between specular and 

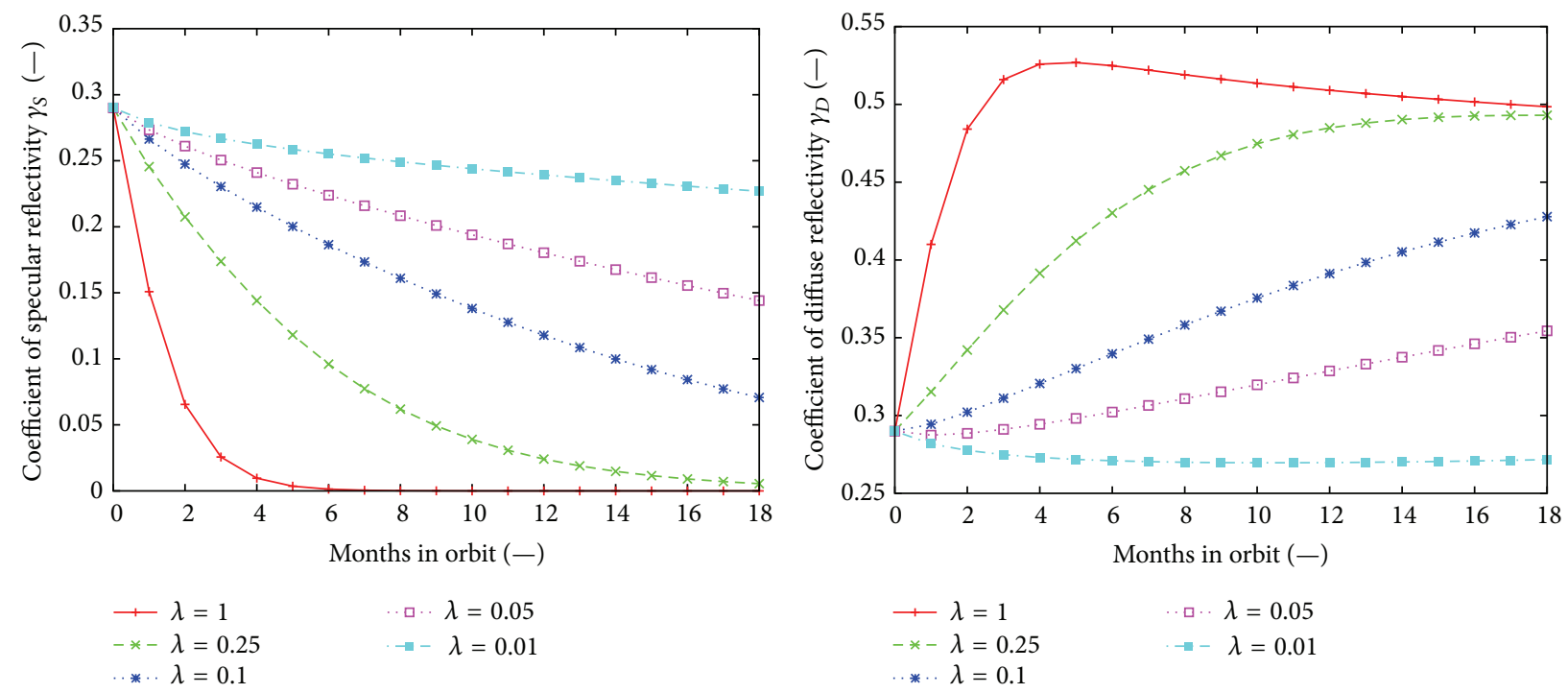

FIGURE 11: Time evolution of $\gamma_{S}$ and $\gamma_{D}$ for different scaling factors. MLI values considered for starting reflectivities with a $\mu_{S D}$ of 1 .

diffuse reflectivities is scaled with an exponential law by the time of duration in orbit:

$$
\mu_{S D}(t)=\frac{\gamma_{S, \mathrm{BOL}}}{\gamma_{D, \mathrm{BOL}}} \cdot e^{-\lambda t}
$$

where $\lambda$ is a scaling factor for the rate of change from specular to diffuse reflectivity. Due to lack of actual data, all polished and metal surfaces are assumed to be nearly perfect specular reflectors at BOL $\left(\mu_{S D}=10\right)$, while MLI is considered to start at a $\mu_{S D}$ of 1 , motivated by the typical crinkled surface structure of MLI. Painted surfaces (such as the rear of the solar panel) also start at a $\mu_{S D}$ of 1 considering a tarnished coating. The EOL/BOL values for specular and diffuse reflectivity as listed in Table 2 are given by

$$
\begin{aligned}
\gamma_{S}(t) & =\mu_{S D}(t) \cdot \gamma_{D}(t), \\
1-\alpha(t) & =\gamma_{S}(t)+\gamma_{D}(t) .
\end{aligned}
$$

Thus, the coefficients of reflectivity evolve to

$$
\begin{aligned}
& \gamma_{S}(t)=(1-\alpha) \frac{\mu_{S D}(t)}{\mu_{S D}+1}, \\
& \gamma_{D}(t)=\frac{1-\alpha}{1+\mu_{S D}(t)} .
\end{aligned}
$$

The scaling factor $\lambda$ is now used to model a faster or slower changing ratio between specular and diffuse reflections. As an example the evolution of diffuse and specular reflectivity for the MLI values specified in Table 2 is displayed in Figure 11.

For the MICROSCOPE case a moderate change towards diffuse reflection is considered. Therefore, a scaling factor of $\lambda=0.1$ has been chosen to obtain the EOL values of diffuse and specular reflectivity as listed in Table 2. Figure 12 shows the evolution of the coefficient of absorptivity for external components subjected to degradation following the model described in this section. The assignment of material models

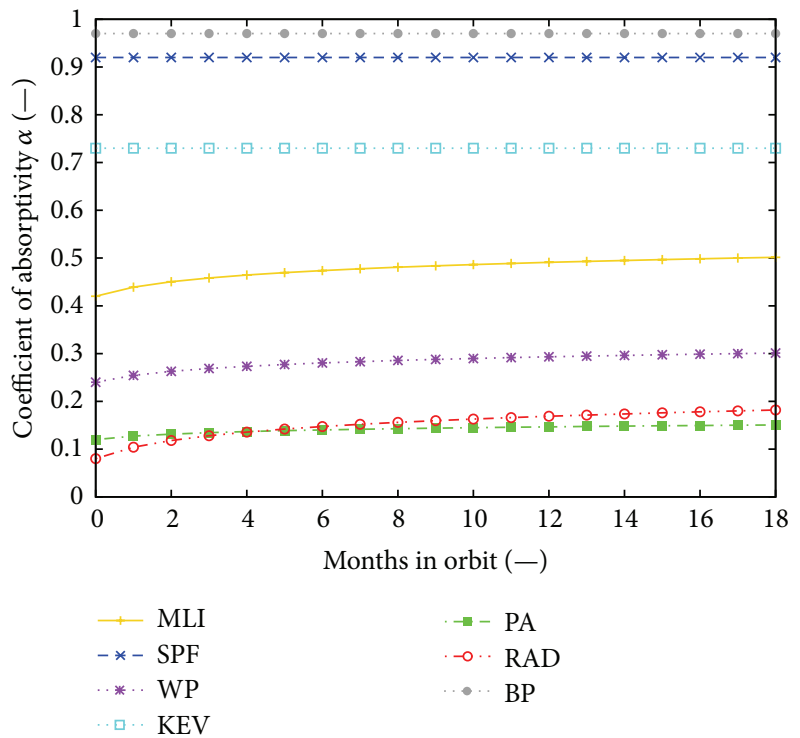

FIGURE 12: Considered variation of solar absorptivity over mission lifetime of 18 months for chosen external components of MICROSCOPE test case. MLI: Multilayer Insulation, SPF: Solar Panel Front, WP: White Paint, KV: Kevlar, PA: Polished Aluminum, RAD: Radiator surface, and BP: Black Paint.

to individual components is depicted in Figure 5. Figure 13 shows the resulting evolution of diffuse and specular reflectivity for $\lambda$ and $\mu_{S D}$ values as discussed above. As a consequence of this reflection model, a decrease of SRP force over time can be expected following (5).

However, one has to keep in mind that the actual illumination condition also affects the resulting force magnitude. Thus, a change of satellite attitude and position over time may lead to a different trend.

The resulting SRP force is calculated with a time resolution of 1 month. Here the position of the MICROSCOPE 


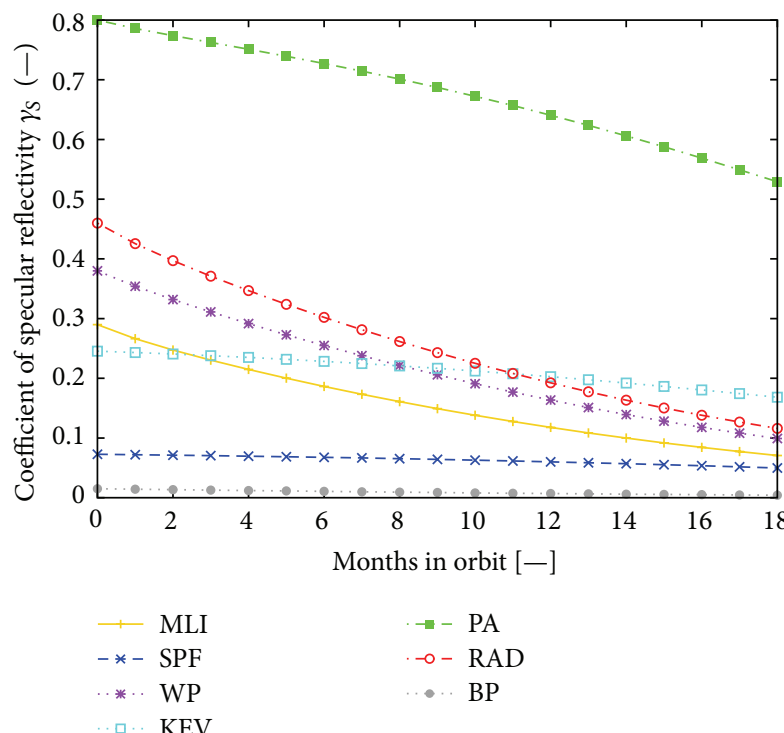

(a)

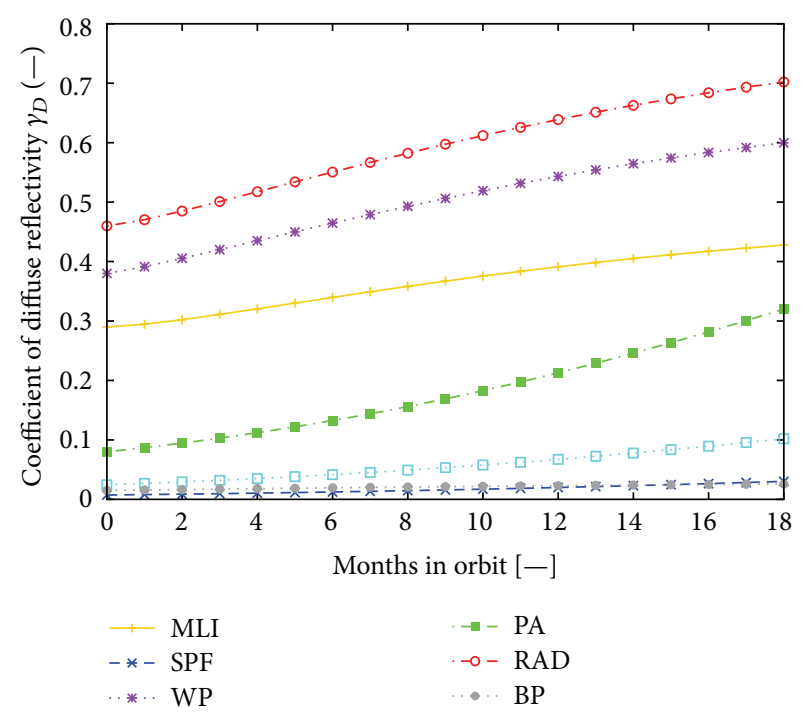

(b)

FIGURE 13: Considered variation of specular (a) and diffuse (b) reflectivity over mission lifetime of 18 months for chosen external components of MICROSCOPE test case. MLI: Multilayer Insulation, SPF: Solar Panel Front, WP: White Paint, KV: Kevlar, PA: Polished Aluminum, RAD: Radiator surface, and BP: Black Paint.

spacecraft with respect to Sun and Earth as well as the spacecraft attitude is fixed; that is, $P$ and $\vec{e}_{\text {Sun }}$ are constant for each investigated case.

Figure 14 shows the obtained results. Again, perpendicular solar irradiation for each satellite side and illumination conditions estimated for MICROSCOPE were chosen. The picture in the center of Figure 14 shows that the force decreases and deviates about 5\% compared to the BOL value for the solar panel side $+X$. For all other sides the effect is even stronger. This is due to the fact that the degradation of the solar panels which are the main contributors of side $+X$ is small compared to all other materials. At the bottom of Figure 14 one can see that the degradation effect is less strong for combinations of $\varphi$ and $\theta$ that form a deviation of $15^{\circ}$ from the normal axis of $+X$ compared to the result for normal incident sunlight. For deviations about $30^{\circ}$, the force even increases during lifetime. In all cases different sides of the satellite contribute to the value of the SRP force whereas the dominating effect of the solar panels decreases with increasing deviation from the $+X$ normal axis.

Finally, in Figure 15 the degradation effect is applied to the MICROSCOPE scenario for the FE model. For comparison the evolution of the force without degradation is also depicted (cf. Figure 9). The value of the force including material degradation was only evaluated at one point per month because an integrated degradation algorithm in the simulation process has not been implemented so far. Nevertheless, the figure shows that omitting the degradation effect will lead to an over- or underestimation of the actual SRP force.

4.5. Benefits for MICROSCOPE. The analysis presented here shows that a thorough assessment of the influence of SRP is highly relevant with respect to the main scientific goal of MICROSCOPE. The goal of the mission is to detect a differential acceleration signal at the orbit frequency $f_{\text {orbit }}$ in the inertial pointing mode which would imply a violation of the EP. At the targeted accuracy of the evaluation of the possible EP violation $\left(10^{-15}\right)$, the science data has to be cleaned from residual accelerations larger then $10^{-12} \mathrm{~m} / \mathrm{s}^{2}$, especially at the frequency $f_{\text {orbit }}$. In order to realize this, MICROSCOPE's AOCS is based on a drag-free concept which keeps the spacecraft in the favored state. However, one has to consider several effects that may lead to external disturbances influencing the internal inertial sensors, regardless of the drag-free control. On the one hand, time delays in controller and actuator responses may cause an influence of external disturbances on the science signal when the satellite state changes with a high rate (as in tumbling or when the satellite enters/leaves eclipse). On the other hand, the inertial sensors are not completely decoupled from external accelerations since misalignments and different response times of the sensor components cause residual internal accelerations affecting the measurement in the range of the orbit frequency. Since the magnitude of the SRP force is in the range of $10^{-5} \mathrm{~N}$ which results in accelerations of about $10^{-8} \mathrm{~m} / \mathrm{s}^{2}$, residual effects on the science signal cannot be neglected completely. Any SRP residual effect will show up at a frequency of $\left(f_{\text {orbit }}+\Delta f\right)$, where $\Delta f$ is a phase difference caused by the Earth's orbit around the Sun. As a consequence, it might be possible to mitigate this influence by analysing a long time span of science data. However, this is subject to further investigation.

Apart from these considerations regarding the later scientific data analysis, a detailed modelling of the corresponding SRP forces and torques is also important to estimate the actual needed control forces of the AOCS for an evaluation 


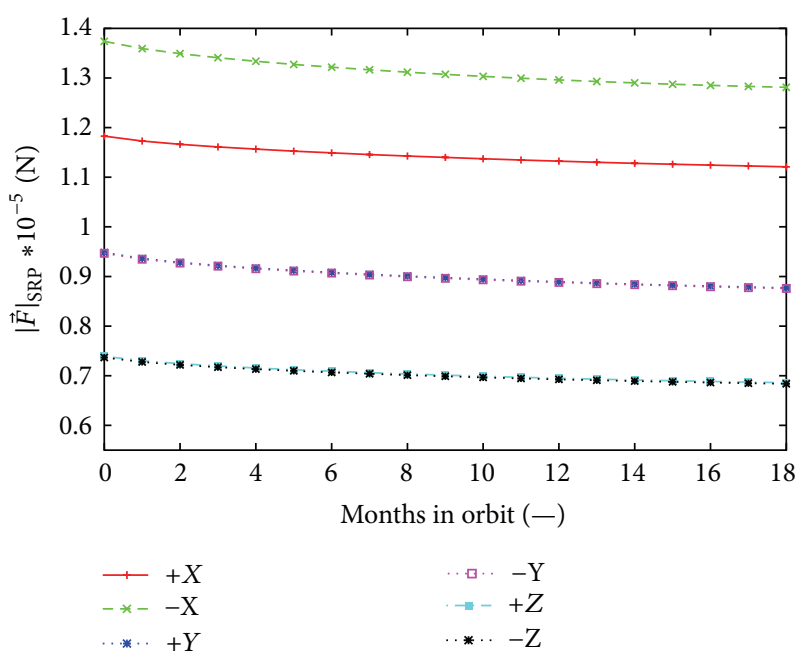

(a)

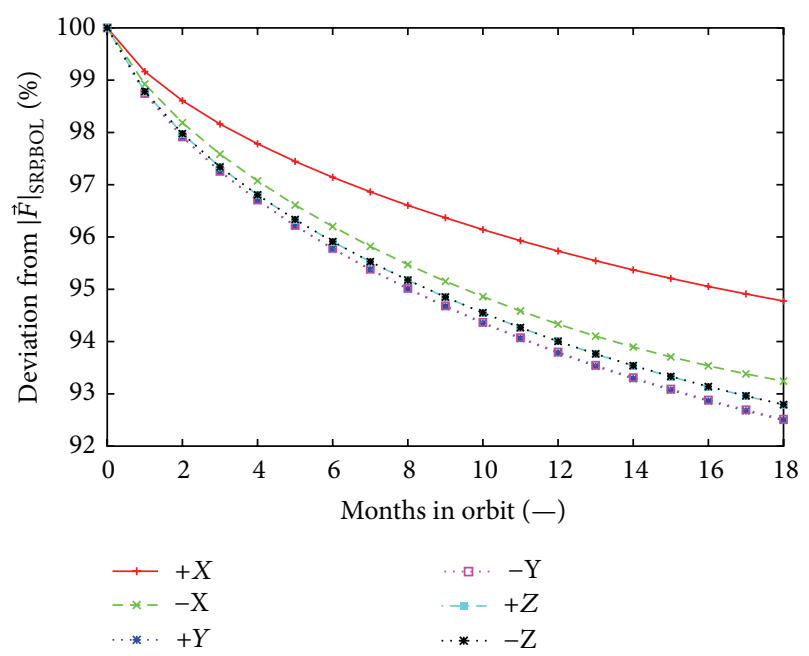

(b)

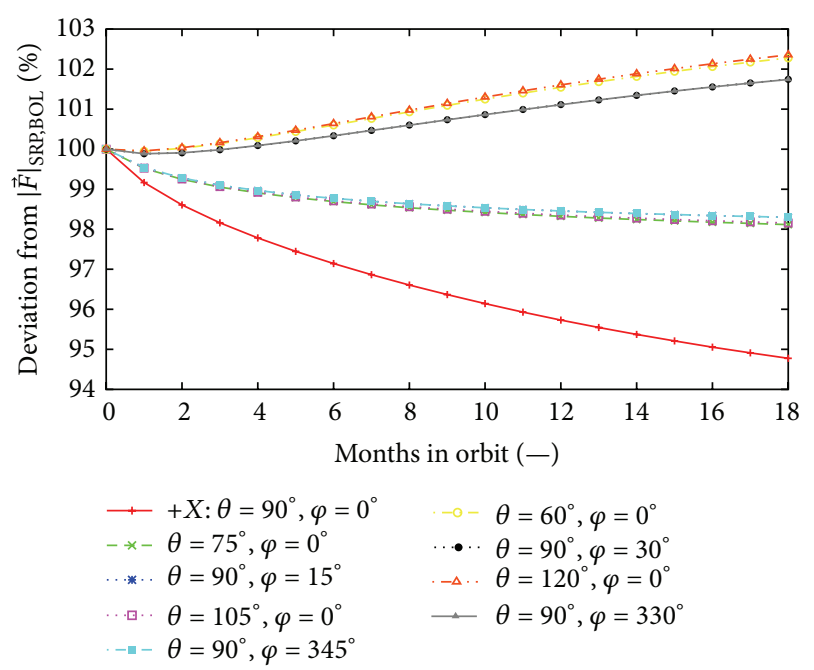

(c)

FIGURE 14: Influence of material degradation on disturbance force due to SRP. (a) Absolute value for perpendicular solar irradiation of each satellite side. (b) Corresponding percentage deviation from BOL value. (c) Percentage deviation from BOL value for illumination conditions that are estimated for MICROSCOPE.

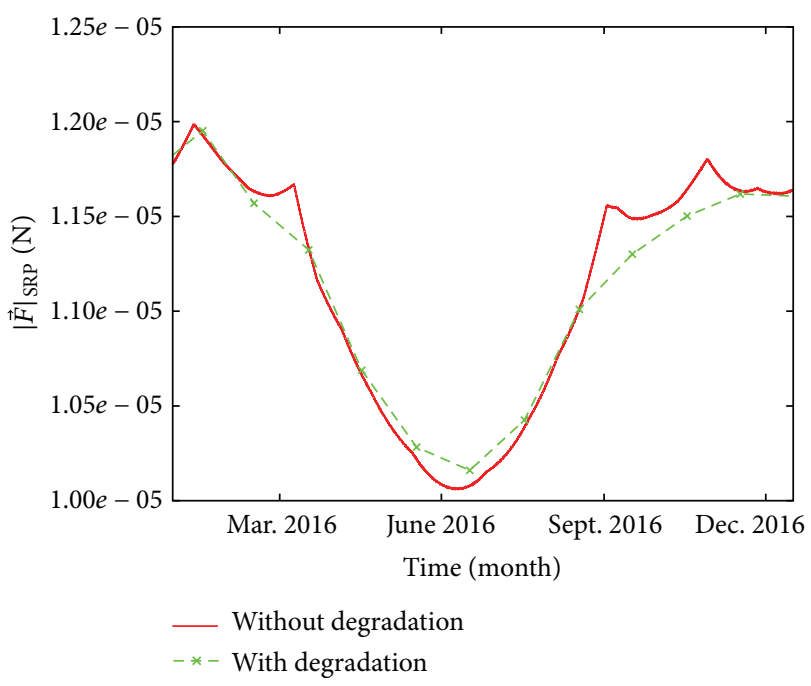

FIGURE 15: Comparison: $|\vec{F}|_{\text {SRP }}$ for the FE model with and without degradation for the MICROSCOPE scenario.

of its performance. The analysis procedure described in this paper reveals explicitly that an incomplete information on the SRP disturbance effect only allows for the identification of its frequency. But an additional determination of its magnitude fails if no effort is put into detailed surface modelling. Both pieces of information are needed to obtain a good knowledge of the actual satellite state and thus provide the possibility of, for example, taking into account cross-coupling effects between the sensors due to residual accelerations. As a consequence, the resulting disturbances due to the here studied effects cannot be neglected at the desired level of MICROSCOPE measurement accuracy.

\section{Conclusion}

The modelling of a realistic disturbance force due to SRP is a complex task which involves a multitude of modelling and simulation steps. In our study we used an algorithm for computing the SRP force which utilises a FE model for estimating the satellite's dimensions and surface properties instead of commonly used Cannonball or Wing-Box models. This algorithm is embedded in the simulation software HPS that amongst others propagates the satellite orbit and attitude. Motivated by the high requirements for attitude precision, the MICROSCOPE mission served as example for the parameter analysis of the different contributors to the SRP force. This study reveals that the analysed NGD cannot be neglected at the desired level of MICROSCOPE measurement accuracy. For this mission case example, the magnitude of $P_{\mathrm{SRP}}$ varies throughout the year about $7 \%$ which is a typical value for many satellite missions. For comparison different simple geometry models of MICROSCOPE were used in addition to the FE model. It was shown that the resulting SRP force varies due to the yearly changes of the magnitude of the SRP pressure $P_{\text {SRP }}$ which were mentioned in this paragraph before. Furthermore, it was shown that a second effect appears which depends strongly (i) on the geometry model of choice and 
(ii) on the satellite's orientation. This second effect is much stronger than the impact of the yearly variation of $P_{\mathrm{SRP}}$. In case of MICROSCOPE, the difference between Solar Panel Front and rear, for example, amounts to approximately $13 \%$ for the FE model. Although the satellite's solar panel will always point to Sun direction, the incident angle will change during the year which yields to variations of the SRP force of at least 9\%. Combining the results for SRP and the FE model a difference of $16 \%$ in the magnitude of the SRP force can be expected for MICROSCOPE over one year. The comparison with other geometry models revealed that from the range of "simple" approaches a disk approach resembles the results of the FE model the most. Another point of the study was the influence of the material degradation. It was shown that for the solar panel side the force decreases and deviates about $5 \%$ from the BOL value at the end of the mission. Depending on the incident angle the degradation effect can even result in an increasing force over time. In summary the study reveals that a simple answer to the question of the main contributor of SRP force cannot be given easily but depends on the actual mission scenario. However, the introduced SRP modelling approach based on FE models enables the highest modelling precision compared to conventional approaches but also implies a considerably high modelling effort. Therefore, the best approach shall be chosen by means of a trade-off between needed SRP force accuracy and resources at hand.

\section{Conflict of Interests}

The authors declare that there is no conflict of interests regarding the publication of this paper.

\section{Acknowledgments}

The authors like to thank their colleagues in the MICROSCOPE teams from ONERA, from Observatoire de la Côte d'Azur, and from CNES for their support. Additionally the authors like to thank thier colleagues René Schwarz and Stephan Theil from the DLR Institute of Space Systems for the fruitful cooperation on the HPS tool. This work is supported by the German Space Agency of DLR with funds of the BMWi (FKZ 50 OY 1305) and by the Deutsche Forschungsgemeinschaft DFG (LA 905/12-1).

\section{References}

[1] J. McMahon, "Improving orbit determination with noncannonball solar radiation pressure models," in Proceedings of the AAS/AIAA Astrodynamics Specialist Conference, Hilton Head Island, SC, USA, August 2013.

[2] J. W. McMahon and D. J. Scheeres, "Improving space object catalog maintenance through advances in solar radiation pressure modeling," Journal of Guidance, Control, and Dynamics, vol. 38, no. 8, pp. 1366-1381, 2015.

[3] B. Rievers and C. Lämmerzahl, "High precision thermal modeling of complex systems with application to the flyby and Pioneer anomaly," Annalen der Physik, vol. 523, no. 6, pp. 439-449, 2011.

[4] J. W. McMahon and D. J. Scheeres, "New solar radiation pressure force model for navigation," Journal of Guidance, Control, and Dynamics, vol. 33, no. 5, pp. 1418-1428, 2010.
[5] O. Montenbruck, U. Hugentobler, R. Dach, P. Steigenberger, and A. Hauschild, "Apparent clock variations of the block IIF-1 (SVN62) GPS satellite," GPS Solutions, vol. 16, no. 3, pp. 303-313, 2012.

[6] O. Montenbruck, P. Steigenberger, and U. Hugentobler, "Enhanced solar radiation pressure modeling for Galileo satellites," Journal of Geodesy, vol. 89, no. 3, pp. 283-297, 2015.

[7] J. Bergé, P. Touboul, and M. Rodrigues, "Status of MICROSCOPE, a mission to test the equivalence principle in space," Journal of Physics: Conference Series, vol. 610, Article ID 012009, 2015.

[8] P. Touboul, G. Métris, V. Lebat, and A. Robert, “The MICROSCOPE experiment, ready for the in-orbit test of the equivalence principle," Classical and Quantum Gravity, vol. 29, no. 18, Article ID 184010, 2012.

[9] M. List, S. Bremer, and C. Lämmerzahl, "Adaption of HPS to the MICROSCOPE mission," in Proceedings of the 62nd International Astronautical Congress, Cape Town, South Africa, 2011.

[10] J. R. Wertz, Ed., Spacecraft Attitude Determination and Control, Kluwer Academic Publishers, Dordrecht, The Netherlands, 1978.

[11] S. Bremer, M. List, H. Selig, H. Rath, and H. Dittus, "Modelling and simulation of the space mission MICROSCOPE," Acta Astronautica, vol. 68, no. 1-2, pp. 28-33, 2011.

[12] Y.-M. Wang, J. L. Lean, and N. R. Sheeley Jr., "Modeling the sun's magnetic field and irradiance since 1713," The Astrophysical Journal, vol. 625, no. 1, pp. 522-538, 2005.

[13] D. P. Rubincam, "LAGEOS orbit decay due to infrared radiation from earth," Journal of Geophysical Research, vol. 92, no. 2, pp. 1287-1294, 1987.

[14] C. J. Rodriguez-Solano, U. Hugentobler, and P. Steigenberger, "Adjustable box-wing model for solar radiation pressure impacting GPS satellites," Advances in Space Research, vol. 49, no. 7, pp. 1113-1128, 2012.

[15] S. K. R. Miller and B. Banks, "Degradation of spacecraft materials in the space environment," MRS Bulletin, vol. 35, no. 1, pp. 20-24, 2010.

[16] P. Touboul and G. Métris, "MICROSCOPE mission scenario," in Proceedings of the Testing the Equivalence Principle, MICROSCOPE Colloquium II, Palaiseau, France, January 2013.

[17] M. Bach, "MICROSCOPE: mission \& satellite configuration," in Testing the Equivalence Principle, MICROSCOPE Colloquium II, Palaiseau, France, 2013.

[18] C. Ingénierie and E. Bellouard, "Microscope thermal control definition document," Tech. Rep. (MIC-DC-S-1-459-CNS), CNES, 2006. 

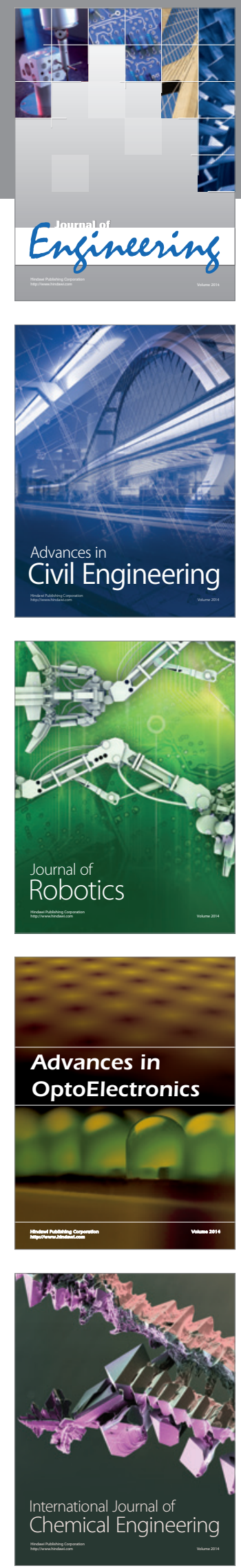

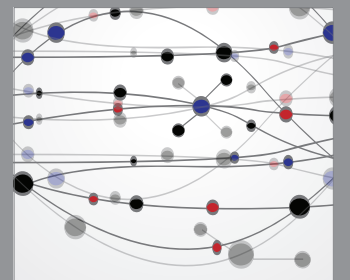

The Scientific World Journal
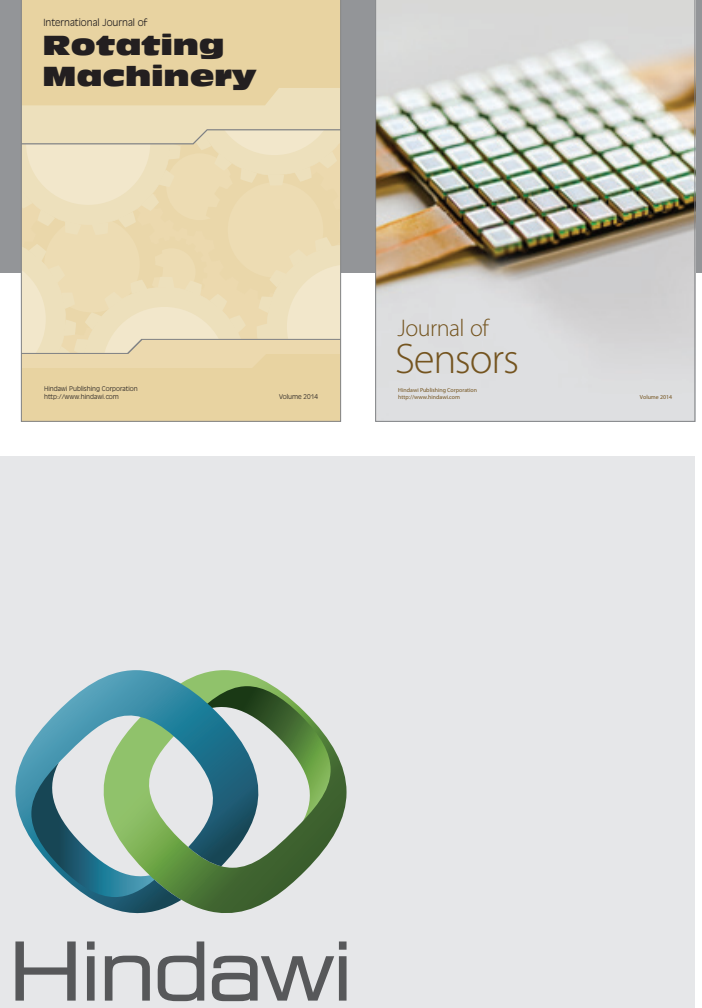

Submit your manuscripts at http://www.hindawi.com
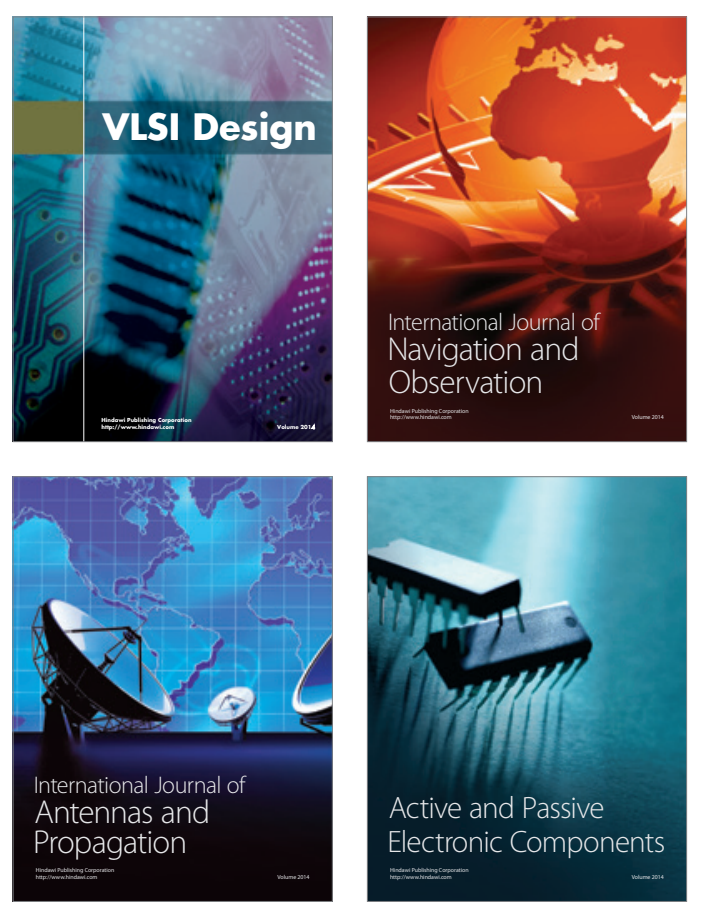
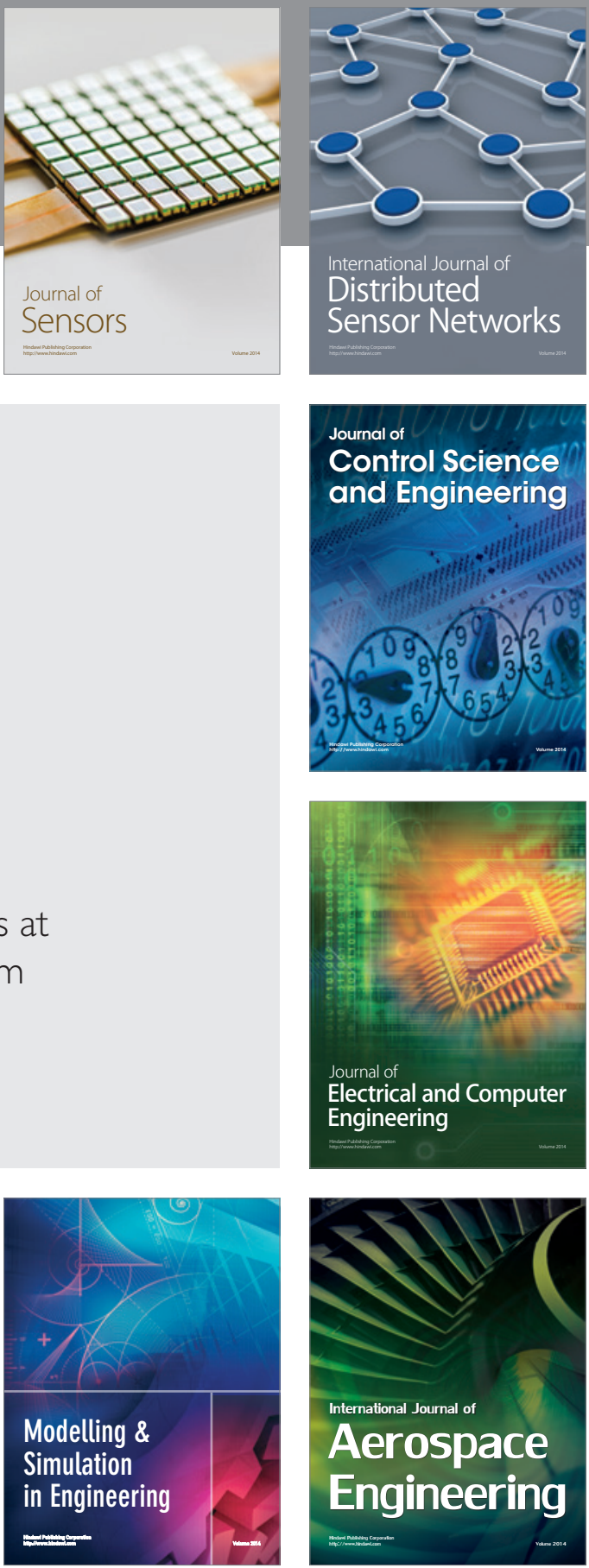

Journal of

Control Science

and Engineering
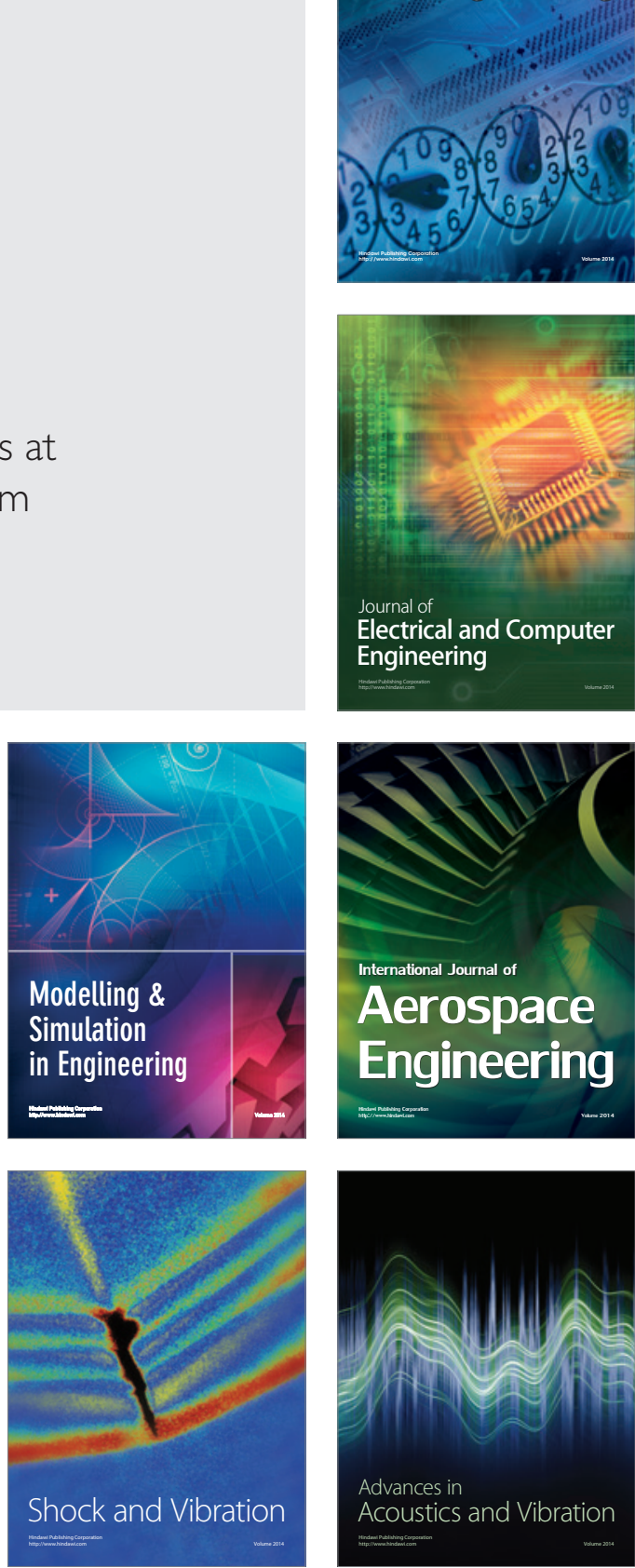